\title{
Numerical Simulations of Capacitive Resistivity Imaging (CRI) Measurements
}

Sebastian Uhlemann (British Geological Survey), Oliver Kuras (British Geological Survey)

suhl@bgs.ac.uk 


\section{Abstract}

Electrical resistivity tomography (ERT) is a well-developed geophysical technique that is used to study a variety of geoscientific problems. In recent years it has been applied to studying permafrost processes at both field and laboratory scale. However, highly resistive surface conditions limit its applicability due to high and variable contact resistances. The use of capacitively coupled sensors is expected to overcome this problem by providing a steady contact impedance regime. Although the theory of capacitive resistivity imaging (CRI) is well understood, a point-pole approximation of the sensors is typically used to show the similarity between CRI and ERT. Due to their nature, capacitive sensors cannot be designed as point-poles as they require a finite extent. This paper assesses the effects the finite size of sensors has on the applicability of CRI theory and aims to provide an improved understanding of the measured data. We employ finite-element numerical modelling to simulate CRI measurements over a homogeneous halfspace and on a finite rock sample. The results of a parameter study over a homogeneous halfspace are compared to an analytical solution. Observed discrepancies between the two solutions clearly indicate that large sensor sizes and small sensor separations violate the point-pole assumption of the analytical solution. In terms of data interpretation, this dictates that sensor separations smaller than twice the sensor size have to be avoided in order to remain below a generic error threshold of 5\%. We show that sensor elevation, halfspace resistivity, halfspace permittivity, and measurement frequency have only minor effects on the discrepancy between simulation and analytical solution. The simulation of sequential CRI measurements on a finite rock sample suggests that, in line with expectations, the measured signals lie mainly in the $4^{\text {th }}$ quadrant of the complex plane. However, also data with negative geometric factors are observed, which are related to uncommon array. A comparison between simulated and measured data showed very good agreement; it validated the simulations and explained the measured data which was acquired using a prototype multisensor CRI system. We show that a 
comparison of simulated and measured imaginary part of the transfer impedance can be used to assess CRI measurement errors. Our work demonstrates that finite-element numerical modelling of CRI measurements is a valuable tool to define limitations on array design and to assess data quality. 


\section{List of keywords}

Finite-element numerical modelling, capacitive resistivity imaging, resistivity tomography, COMSOL, Permafrost 


\section{Introduction}

Electrical resistivity tomography (ERT) is a well-developed and widely used tool applicable to a broad range of problems in near surface geophysics. In recent years it has also been applied to the investigation of permafrost at both field and laboratory scales (Krautblatter and Hauck, 2007; Krautblatter et al., 2010; Kuras et al., 2012). However, the utility of galvanic sensors in permafrost research (or more generally, environments with highly resistive surface conditions) is limited by high levels of, and large variations in, contact resistances between the sensors and the host material. Although a capacitively coupled system using non-grounded electrical dipoles cannot have lower contact impedances than galvanically coupled sensors of the same type (Hördt et al., 2013), it is expected that the capacitive approach will limit the impact of electrode surface degradation (e.g. corrosion) on the quality of contact and provide more stable contact impedances over time. This should enable us to obtain high-quality resistivity measurements, whilst emulating conventional ERT measurements in terms of data processing and interpretation (Kuras et al., 2006).

The theory of capacitively coupled resistivity measurements is well described (Grard, 1990; Tabbagh et al., 1993; Kuras et al., 2006), but typically based on a point-pole approximation of capacitive sensors. We focus here on laboratory studies where the rock samples have to be of finite size, whereas the capacitive sensors cannot be infinitely small and the sensor distribution must be dense to investigate structures of interest with sufficient resolution (Kuras et al., 2012). Therefore, the point-pole approximation is in danger of being violated and the geometric dimensions of the dipole configurations have to be considered.

In this paper we employ finite-element numerical modelling using Comsol Multiphysics $^{\circledR}$ to investigate the effects of using capacitive sensors of finite size compared to the 
standard point-pole approximation. The aim of this study is to determine limitations on array design, to improve our understanding of the response of a recently developed multi-sensor CRI system, and to validate the use of conventional ERT interpretation tools for CRI data. This will be achieved by the comparison of simulated data with analytical solutions and real laboratory measurements.

The parameters chosen for the numerical simulations (i.e. sensor separation and height, sample size, measurement frequencies, and sample resistivities) are derived from typical values encountered in laboratory studies on permafrost affected bedrock, as described by Kuras et al. (2012).

\section{Analytical solution using a quasi-static approach}

CRI is a low-frequency electromagnetic measurement that operates in a quasi-static regime, under which inductive effects, as well as displacement currents or polarization effects, are neglected. This requires the induction number $B$ (a measure of the spatial scale in relation to the skin depth $\delta$ (McNeill, 1980)) to be small, i.e. $\mathrm{B}^{2}$ «1(Benderitter et al., 1994), where $B$ is defined as

$$
B=\frac{l}{\delta}=l \sqrt{\frac{\pi \mu_{0} f}{\rho}}
$$

with the sensor separation $l$, the magnetic permeability $\mu$, the measurement frequency $f$, and the halfspace resistivity $\rho$. For the frequencies $(10-150 \mathrm{kHz})$, measurement scales (below $1 \mathrm{~m})$, and sample resistivities $\left(10^{1}-10^{5} \Omega \mathrm{m}\right)$ used in our laboratory investigations, the induction number tends to be below 1 and hence the condition is likely to be satisfied under typical circumstances (see Table 1). Nevertheless, there is concern that induction effects may become significant at the upper end of the frequency range, or for more conductive materials. 
Based on these assumptions the transfer impedance of a capacitively coupled quadrupole (Figure 1), comprising two poles for current injection $\left(\mathrm{C}_{1}\right.$ and $\left.\mathrm{C}_{2}\right)$ and two for the measurement of a potential difference $\left(\mathrm{P}_{1}\right.$ and $\left.\mathrm{P}_{2}\right)$, can be derived using the method of image sources (Grard and Tabbagh, 1991). It can then be shown that the complex transfer impedance is (Kuras et al., 2006)

$$
Z=\frac{1}{i \omega C_{0}}\left(1-K^{E S} \alpha\right)=Z_{0}\left(1-K^{E S} \alpha\right)
$$

This expression describes the transfer impedance measured by the system as a modification of its free-space impedance $Z_{0}$ (which depends on the mutual capacitance of the sensors $C_{0}$ and the measurement frequency $\omega$ ) by an electrostatic geometric factor $K^{E S}$, comprising the distances between source and receiver points $r$ and image source and receiver points $r$ ':

$$
K^{E S}=\frac{\frac{1}{r r_{11}}+\frac{1}{r r_{2 n}}-\frac{1}{r r_{12}}-\frac{1}{r r_{21}}}{\frac{1}{r_{11}}+\frac{1}{r_{22}}-\frac{1}{r_{12}} \frac{1}{r_{21}}},
$$

and a factor $\alpha$ that comprises the dielectric properties of the media above and below the ground surface. For an air-ground interface this factor $\alpha$ is given by (Kuras et al., 2006):

$$
\alpha=\frac{\rho \omega \epsilon_{0}\left(\epsilon_{r}-1\right)-i}{\rho \omega \epsilon_{0}\left(\epsilon_{r}+1\right)-i},
$$

with the measurement frequency $\omega$, resistivity $\rho$ and relative permittivity $\varepsilon_{r}$ of the ground.

Similarly to conventional dc theory and following Kuras et al. (2006), we can derive an expression for the apparent resistivity, which is given by:

$$
\rho_{a}=-\frac{1}{2 \omega \varepsilon_{0}}\left(\frac{\left(K^{E S}-1\right)^{2} K^{E S}+C_{0}^{2} \omega^{2} Z^{2}}{-C_{0} \omega Z K^{E S}}\right) .
$$


For practical CRI measurements the electrostatic geometric factor $K^{E S}$ is usually assumed to be approximately equal to one (note, $K^{E S}=1$ implies that sensors are located at the ground surface) and the measured potential is expected to be approximately in-phase with the injected current. Therefore, equation (5) simplifies to

$$
\rho_{a} \approx \frac{c_{0}}{2 \epsilon_{0}} \operatorname{Re}(Z)
$$

where the mutual capacitance $C_{0}$ is given as

$$
C_{0}=\frac{4 \pi \varepsilon_{0}}{\frac{1}{r_{11}}+\frac{1}{r_{22}}-\frac{1}{r_{12}}-\frac{1}{r_{21}}}
$$

Inserting equation (7) into (6) the apparent resistivity becomes

$$
\rho_{a} \approx \operatorname{Re}(Z) \cdot K^{d c}=\frac{\operatorname{Re}(\Delta V)}{\hat{I}} K^{d c},
$$

with the measured complex potential difference $\Delta V$, the amplitude of the injected current $\hat{I}$, and the dc geometric factor $K^{d c}$, which can be defined in terms of the sensor separation $l$ as (Reynolds, 2011)

$$
K^{d e}=\pi l(2+\sqrt{2}) .
$$

Clearly, equation (8) is an analogue of the dc expression for the apparent resistivity. This implies that, according to the quasi-static theory, the real part of the complex transfer impedance can be used to estimate a dc apparent resistivity.

This theoretical formulation suggests that under certain conditions (essentially the use of point poles on the surface within the quasi-static regime) CRI is capable of emulating ERT measurements, without the need for galvanic coupling to the ground. This provides the opportunity to use well-established ERT inversion routines to estimate models of the resistivity distribution of specific target structures. 
This theoretical framework leaves a number of practical questions unanswered. For practical, finite-size capacitive sensor geometries, limits must be defined where the point pole approximation remains valid. Moreover, the effects of ground dielectric properties on the measured transfer impedance of a practical quadrupole must be studied. Our investigation will compare numerical simulations to the point pole formulation. In the first instance therefore, the dependence of the analytical solution on its geometric properties have to be understood.

Kuras et al. (2006) presented an extensive study of the effects of parameter variations on the transfer impedance, which shows that for sufficiently large resistivities $(\rho>1,000 \Omega \mathrm{m})$ the real part of the complex transfer impedance is practically equal to its corresponding $\mathrm{dc}$ value, independent of the sensor separations. However, it was also shown that the transfer impedance is more sensitive to sensor elevation than separation, particularly in its imaginary component. Figure 2 shows the behaviour of the real and imaginary parts of the complex transfer impedance with increasing sensor elevation for a square unit array. For a realistic range of elevations, the real part is clearly less affected by the sensor elevation than the imaginary part. Up to elevations of about $0.1 \mathrm{~m}$, the real part is constant and equal to the dc solution for all resistivities under investigation. Above 0.1 m elevation, $\operatorname{Re}(\mathrm{Z})$ decreases monotonically, independent of the ground resistivity. $\operatorname{Im}(Z)$ is clearly resistivity dependent and shows stronger dependence on the sensor elevation (i.e. it varies over 5 orders of magnitude). Over resistive ground $(\rho>1,000 \Omega \mathrm{m})$ the imaginary part remains constant for a wider range of small sensor elevations (up to $\sim 1 \mathrm{~mm}$ ). The observed behaviour of the real and imaginary components shows that the real part of $Z$ in the capacitive case can be compared with the magnitude of $Z$ for an equivalent dc array, provided that sensor elevations are small. The behaviour is also independent of sensor separation, which suggests that the use of standard dc data processing tools is justified. Assessment of the imaginary part, especially for conductive media, is likely to be of limited value due to its strong dependency on the sensor geometry. In the following 
numerical simulations, the analytical formulation (hereafter denoted PP) will be used as a reference for appraising the effects of sensors of finite size.

\section{Numerical Simulation of CRI Measurements}

Forward modelling of geophysical data is usually done for two reasons: Firstly, to develop an improved understanding of the physical processes taking place during a measurement, and secondly, as a tool to interpret measured data (Butler and Sinha, 2012). Both goals will be pursued in this paper. Parameter studies over a homogeneous halfspace are intended to provide deeper insight into the physical processes; the results lead to a definition of limitations on array design and a better understanding of the effects on the complex transfer impedance of subsurface properties and measurement parameters. Finally, a comparison of simulated and measured data tests the agreement between simulation and reality.

For the following numerical simulations we have used Comsol Multiphysics ${ }^{\circledR}$, which provides a generic finite-element $(\mathrm{FE})$ modelling environment. FE with unstructured grids is beneficial in the simulation of CRI measurements due to the significant differences in length scales (i.e. sensor separation $\sim 1 \mathrm{~m}$, sensor elevation $\sim 1 \mathrm{~mm}$ ), where a discretisation using regular grids would lead to a large number of cells. FE has the ability to adopt to arbitrary geometries and as a consequence no geometrical perturbations of the electrical field are introduced (Marescot et al., 2006).

Nevertheless, the large contrast in length scales for CRI still requires careful mesh design and typically leads to a relatively complex model parameterisation. Together with the underlying physical relationships (quasi-static approximation), modelling of CRI measurements is a non-trivial exercise where care has to be taken to achieve a satisfactory convergence and reasonable model solutions. For the numerical simulations we take advantage of the AC/DC module of Comsol Multiphysics ${ }^{\circledR}$, where a quasi-static regime is assumed in the same way as described above for the analytical PP formulation. 


\section{Homogeneous Halfspace}

In order to compare the performance of capacitive sensors with finite dimensions (henceforth denoted FS) to that of point electrodes, a quasi-static quadrupole of square plate electrodes is modelled over a homogeneous halfspace using a range of parameters for its geometrical configuration (i.e. sensor size, separation, and elevation) and measurement conditions (i.e. halfspace resistivity, halfspace permittivity, measurement frequency). The results can then be compared to the analytical PP solution.

The FS model consists of two blocks representing ground overlain by air with dimensions $30 \mathrm{~m} \times 30 \mathrm{~m} \times 25 \mathrm{~m}$ and $30 \mathrm{~m} \times 30 \mathrm{~m} \times 15 \mathrm{~m}$, respectively. The quadrupole is modelled as an equatorial square array (i.e. equal sensor separation in every direction) and is placed within the upper air halfspace; air is also used as a dielectric between sensors and ground. The large dimensions of the model domain compared to the sensor separation (max. 1 m) were chosen so as to minimise boundary effects. Moreover, infinite elements were assigned to the outer boundaries, where the potential field is extended to infinity (COMSOL, 2011). These two approaches provide a good approximation of the usual assumption of infinite dimensions within geophysical problems (Butler and Sinha, 2012). Dirichlet boundary conditions $(V=0)$ have been assigned to the outermost boundaries of the model domain. Current electrodes are modelled using a so-called "terminal"-type boundary condition, which simulates a connection to an external electric circuit through which a specific voltage or current can be applied to the electrodes. This method has the advantage that the electrode domains are intrinsically modelled as perfect conductors, without having to assign infinitely small resistivity (COMSOL, 2011). This implementation avoids strong resistivity contrasts between sensors and surroundings and thus contributes to a well posed problem. For the injection dipole, sensor domains are homogeneously charged with an injection current of $I_{0}=1 \mathrm{~mA}$; opposing polarity is assigned to the two poles to ensure current flow between the sensors. Figure 3 shows the configuration as implemented in Comsol together with an example mesh. 
In the following, the discrepancies between FS and PP will be expressed as relative errors, defined as their difference normalized by the PP solution:

$$
\text { relative error }(\%)=\frac{F S-P p}{P P} \cdot 100 .
$$

We have to emphasize here that this formulation may incorporate numerical errors (e.g. due to mesh discretisation and numerical inaccuracy), as well as violations of the PP approximation (i.e. use of point poles, low induction numbers), given the chosen model parameters. In order to minimise the influence of numerical inaccuracies, for most of the models the mesh parameters (i.e. maximum cell size, grow factor, and edge resolution) have been adapted to reflect changes in the sensor size and elevation. Additionally, basic numerical checks (e.g. comparing solutions on different meshes) have been carried out.

Sensor separation vs halfspace resistivity. A crucial parameter for experimental array design is the minimum separation between adjacent capacitive sensors. We therefore examine normalised sensor separation $l_{r}$, defined as the absolute sensor separation $l$ normalised by the sensor size $w$ (i.e. the side length of a square plate sensor).

$$
l_{r}=\frac{l}{w}
$$

The top panel of Figure 4 shows the absolute values of the transfer impedance $Z$ (as obtained from the PP solution) for a relevant subset of the parameter space (i.e. realistic combinations of normalised sensor separation and halfspace resistivities, with values ranging from $1.1 \mathrm{~m} / \mathrm{m}$ to $20 \mathrm{~m} / \mathrm{m}$ and from 100 to $100,000 \Omega \mathrm{m}$, respectively). Real and imaginary components of $Z$ show similar behaviour; as expected, increasing halfspace resistivities result in increasing complex transfer impedances. Increasing sensor separations (corresponding to increasing geometric factors) on the other hand lead to decreasing $\mathrm{Z}$. The real part is more sensitive to halfspace resistivity than to sensor separation. The imaginary part shows the same 
sensitivity for halfspace resistivities above $1,000 \Omega \mathrm{m}$, while below the absolute value of the imaginary part of $Z$ is more sensitive to the sensor separation.

The lower panel of Figure 4 shows the normalised difference between FS and PP ("relative error"). The real part shows the expected behaviour; for large sensor separations the solutions for the two approaches (i.e. FS and PP) are almost identical and the relative errors small $(<0.1$ $\%$ ), whereas for smaller relative separations $\left(l_{r}<7\right)$ an exponentially increasing deviation (up to $20 \%$ and more) with decreasing distance can be observed. These results suggest that for very small sensor separations $\left(l_{r} \leq 2\right)$ the PP approximation is violated. Large discrepancies between FS and PP solutions imply that the fundamental linear relationship between measured real part of $\mathrm{Z}$ and apparent resistivity (see eq. 8) is compromised, since this holds for point poles only. Therefore, in order to justify the use of conventional ERT methods for interpretation of CRI measurements, the deviation due to the use of finite-size sensors should remain below an acceptable error threshold, here we choose $5 \%$. This implies that measurements using sensor separations smaller than twice the sensor size (i.e. $l_{r}<2$ ) should be avoided. The halfspace resistivity is shown to have only minor effects on the previously described behaviour. Only for very large resistivities of $\rho>100 \mathrm{k} \Omega \mathrm{m}$ the discrepancy at small separations reduces significantly ( $\sim 8 \%$ compared to $\sim 22 \%$ for smaller resistivities). This suggests improved performance of the capacitive resistivity technique in resistive environments, as highlighted by previous authors (e.g. Tabbagh et al., 1993; Kuras et al., 2006).

A high sensitivity towards sensor separation and halfspace resistivity was found for the relative error of $\operatorname{Im}(\mathrm{Z})$. The relative errors between $\mathrm{FS}$ and $\mathrm{PP}$ solutions vary from $0.1 \%$ to more than $100 \%$ across the parameter space. However, the general behaviour in terms of the sensor separation is comparable to the real part; the smaller the sensor separation, the larger the discrepancy between the simulation and the PP solution. This indicates again the violation of the point pole approximation. In contrast to the real part, the halfspace resistivity has a significant influence on the size of the error; resistivities larger than $10 \mathrm{k} \Omega \mathrm{m}$ show rapidly decreasing 
relative errors with increasing halfspace resistivity. With resistivities as high as $\rho=100 \mathrm{k} \Omega \mathrm{m}$ (e.g. permafrost) and $l_{r}>4$ the error becomes comparable to that of the real part and shows hardly any sensitivity towards the sensor separation, but increases very strongly (i.e. over 2 orders of magnitude) for smaller values of the two parameters. Once more, the suggestion is that CRI is likely to perform better in resistive environments (e.g. permafrost). But due to its high sensitivity to the sensor geometry and halfspace resistivity, care has to be taken when evaluating the imaginary part.

Sensor elevation vs halfspace resistivity. Another important parameter in terms of the sensor geometry is the sensor elevation $h$ above the ground, since it usually cannot be determined exactly in practical experiments (e.g. due to the ground roughness). Whilst in the simulation ideal surface conditions have to be assumed, sensor elevation is varied to account for this uncertainty to some extent (i.e. $h$ ranging from $0.001 \mathrm{~m}$ to $0.075 \mathrm{~m}$ ). The effects of the quadrupole elevation on the transfer impedance and the discrepancy between the FS and PP solutions are shown in Figure 5.

The absolute values of $Z$ (top panel of Figure 5) show similar sensitivities to those described for the sensor separation. Within the chosen range of sensor elevations, the transfer impedance depends mainly on the halfspace resistivity. The imaginary part shows the opposite behaviour; with increasing sensor elevation the imaginary part of $Z$ increases, whereas with increasing halfspace resistivity the sensitivity towards the sensor elevation decreases.

Sensor elevations are incorporated in the analytical solution through the geometric factor $\mathrm{K}^{\mathrm{ES}}$ (see eq. 2 and Figure 2), therefore only minor discrepancies between the real parts of the numerical simulation and analytical solution (lower panel of Figure 5) are found, with variations of the relative error between $0.05 \%$ and $0.45 \%$. Hence, for all elevations under investigation $\operatorname{Re}(\mathrm{Z})$ can be accurately approximated by the analytical PP solution. The variations for different sensor elevations appear to be controlled by the mesh discretisation 
between the lower face of the sensor domain and the surface boundary of the lower halfspace; thus they reflect numerical inaccuracy.

Discrepancies between FS and PP are more pronounced for the imaginary part. For small sensor elevations and halfspace resistivities relative errors are large (up to 100\%), but they are decreasing rapidly with increasing sensor elevation and halfspace resistivity. These results can be explained by two effects. Firstly, a higher sensitivity of the imaginary part to numerical inaccuracies, since absolute values of $\operatorname{Im}(Z)$ are small where relative errors are high. Secondly, the strong growth of $\operatorname{Im}(Z)$ with sensor elevation and halfspace resistivities causes the relative error between FS and PP to be reduced in significance.

Sensor elevation vs sensor separation. Figure 6 shows a sensitivity study of sensor elevation against normalised sensor separation. The absolute values of $\mathrm{Z}$ (top panel) show basically the same behaviour as shown previously. With increasing sensor separation the geometric factor increases and hence the transfer impedance decreases. For small changes of the sensor elevation $(h<0.03 \mathrm{~m}) \operatorname{Re}(Z)$ remains nearly constant for a chosen sensor separation. Much stronger changes can be observed for the imaginary part; both, sensor elevation and separation, affect the transfer impedance and can lead to significant changes in $\operatorname{Im}(Z)$, whereas increasing values for the sensor elevation and decreasing values for the separation result in increasing imaginary parts of the transfer impedance (i.e. over 7 orders of magnitude).

Comparing the FS against the PP solutions (lower panel of Figure 6) shows that the discrepancy within $\operatorname{Re}(Z)$ is more sensitive to changes of the sensor separation than to changes in the sensor elevation. However, specific combinations of sensor elevation and separation exist, at which the discrepancy between FS and PP becomes minimal. These areas might indicate favourable numerical conditions, such as optimal mesh geometry and parameterisation. In contrast to $\operatorname{Re}(Z)$ the relative error of $\operatorname{Im}(Z)$ shows more sensitivity towards the sensor elevation, where small elevations and separations cause the largest error (> $100 \%)$. The large 
error of the imaginary part for small sensor elevations $(h<0.01 \mathrm{~m})$ is independent of the sensor separation, which suggests numerical problems due to suboptimal mesh discretisation.

Additionally, small sensor separations $\left(l_{r}<2\right)$ introduce a larger error for higher elevations $(h<$ $0.025 \mathrm{~m}$ ); this is likely to be caused by the finite size geometry (FS case), which is not accounted for in the PP solution.

Pole elevation vs halfspace resistivity. In practice it cannot be guaranteed that each sensor has exactly the same elevation above ground, as assumed by the quasi-static theory for the derivation of equation 8. Figure 7 shows the discrepancy between FS and PP for the variation of the elevation of one pole (i.e. leaving the remaining three poles at $h=0.001 \mathrm{~m}$ ) at a range of halfspace resistivities. Note, the PP solution was calculated with all four poles at the same elevation. The relative error for $\operatorname{Re}(\mathrm{Z})$ remains small $(<0.1 \%)$ for a difference in pole height of up to $0.05 \mathrm{~m}$, but increases exponentially thereafter. The marginally smaller errors at pole elevations between $0.02 \mathrm{~m}$ and $0.04 \mathrm{~m}$ are likely to be due to mesh effects. As for the previous studies, the imaginary part shows substantially more variability. For halfspace resistivities below $300 \Omega \mathrm{m}$, the error is about $100 \%$ (comparable with Figure 6). As previously discussed, this is likely to be caused by a mesh discretisation that is too coarse between the sensor domain and halfspace, thus causing numerical inaccuracies. Due to the large sensor separation $\left(l_{r}=20\right)$ induction effects can be ruled out here $\left(B=2.04 \cdot 10^{-4}\right)$. However, with resistivities above 300 $\Omega \mathrm{m}$ the relative error of $\operatorname{Im}(\mathrm{Z})$ is decreasing and shows sensitivity towards the pole elevation; increasing pole height leads to an increasing relative error. For resistivities above $50 \mathrm{k} \Omega \mathrm{m}$ the sensitivity towards pole height is negligible and the error for the imaginary part small throughout the investigated elevations.

From a practical point of view, these results show that the overall sensitivity towards sensor elevation (quadrupole elevation, but also pole elevation, which is likely to be less accurately determined) is lower than towards dipole separation. Thus, not every pole of the 
quadrupole has to have exactly the same height above ground to obtain meaningful results and be interpretable with dc theory.

Measurement frequency vs halfspace resistivity. The measurement frequency is an essential parameter of the CRI technique. Figure 8 shows a sensitivity study of the halfspace resistivity for a typical quadrupole (i.e. $h=0.001 \mathrm{~m}, l_{r}=20$ ). For halfspace resistivities smaller than 1 $\mathrm{k} \Omega \mathrm{m}$, the real part of the transfer impedance is independent of the measurement frequency. Also the imaginary part of the transfer impedance shows only minor variations. For halfspace resistivities above $1 \mathrm{k} \Omega \mathrm{m} \operatorname{Re}(\mathrm{Z})$ is decreasing with increasing measurement frequency, whereas $\operatorname{Im}(\mathrm{Z})$ is mainly increasing, but showing a more complex behaviour.

Only small discrepancies between the FS and PP solutions (lower panel of Figure 8) can be found for $\operatorname{Re}(Z) \cdot \operatorname{Im}(Z)$ however shows significantly more variability within the relative errors. Small halfspace resistivities and measurement frequencies $(<1 \mathrm{k} \Omega \mathrm{m}$ and $<400 \mathrm{kHz})$ are associated with large errors (up to $100 \%$ ), which are caused by numerical inaccuracies, as observed previously for the modelled quadrupole configuration. However, these errors reduce to less than $10 \%$ for resistivities above $2 \mathrm{k} \Omega \mathrm{m}$ and frequencies above $30 \mathrm{kHz}$, which are likely to be related to changes in the induction number $B$. Whilst for a small halfspace resistivity and measurement frequency of $10 \Omega \mathrm{m}$ and $10 \mathrm{kHz}$, respectively, the induction number is $B=0.063$, it drops one order of magnitude to $B=0.007$ for a halfspace resistivity of $2 \mathrm{k} \Omega \mathrm{m}$ and a frequency of $30 \mathrm{kHz}$. The correlation of halfspace resistivity and relative error seems to be stronger than for the frequency, since smaller frequencies $(<30 \mathrm{kHz})$ show still large errors at a resistivity of about $2 \mathrm{k} \Omega \mathrm{m}$, albeit decreasing induction numbers; only at resistivities as high as $10 \mathrm{k} \Omega \mathrm{m}$, the relative error has dropped down to acceptable levels. With frequencies above 500 $\mathrm{kHz}$ the relative error is decreasing noticeably, even for small resistivities $(<1 \mathrm{k} \Omega \mathrm{m})$; this is an observation that is independent of the induction number, which would be comparably large at these resistivity-frequency regimes (e.g. $B=0.044$, for $\rho=1 \mathrm{k} \Omega \mathrm{m}$ and $f=500 \mathrm{kHz}$ ) and inductive effects can no longer be neglected. This highlights that, whilst the difference between 
numerical and analytical solution may become small, the assumption of a quasi-static regime may break down and the physics are not correctly represented by the simulation anymore. Note that the largest errors are found for combinations of halfspace resistivity and measurement frequency for which $\operatorname{Im}(Z)$ is smallest, which might indicate inaccuracies within the numerical solution.

These results show that the measurement frequency does not affect the validity of the PP approximations for $\operatorname{Re}(Z)$, and hence irrespective of the chosen frequency $\operatorname{Re}(Z)$ for a CRI measurement should be equal to that of a dc measurement (provided that all other parameters are appropriately chosen). Although the relative error of the imaginary part shows more variability, changes with measurement frequency are only small, but indicate an improving performance towards very high frequencies $(<1 \mathrm{MHz})$. Kuras et al. (2006) define an upper frequency limit for the CRI technique below which inductive effects for point poles can be neglected, which depends on the ground conditions

$$
f<f_{u l}=\frac{\rho}{\pi \mu_{0} l^{2}} .
$$

This upper limit will increase with increasing halfspace resistivity, assuming that the sensor separation $l$ remains constant. For the smallest halfspace resistivity under investigation, i.e. $\rho=10 \Omega \mathrm{m}$, this upper frequency limit is $f_{u l} \approx 2.5 \mathrm{MHz}$, and hence all chosen frequencies are well below this limit. While this limit will apply for small scale applications, e.g. at laboratory experiments, field measurements are usually restricted to an upper frequency limit of $25 \mathrm{kHz}$. However, in the discussion of Figure 8 we saw that this limit might be at least an order of magnitude smaller using sensors with finite dimensions instead of point poles.

Halfspace relative permittivityvs halfspace resistivity. Figure 9 shows the dependence of $Z$ on the halfspace permittivity and resistivity. $\operatorname{Re}(Z)$ and $\operatorname{Im}(Z)$ (upper panel of Figure 9) are strongly dependent on the halfspace resistivity, but show also a minor dependence on the halfspace permittivity (note the similarity to Figure 8). Increasing permittivity results in 
increasing dielectric polarization and in a decreasing real part of the transfer impedance, an effect that is more pronounced at high resistivities $(>10 \mathrm{k} \Omega \mathrm{m})$. The dielectric polarization also changes the phase of the signal, which can be seen at the increasing imaginary part of the transfer impedance at higher halfspace resistivities. This effect is strongest at permittivities between 5 and 10 .

The lower panel of Figure 9 shows the discrepancy between FS and PP solution. $\operatorname{Re}(\mathrm{Z})$ shows a small relative error of about $0.05 \%$ throughout the investigated $\varepsilon-\rho$ space. A larger variability can be seen for $\operatorname{Im}(\mathrm{Z})$. For halfspace resistivities larger than $5 \mathrm{k} \Omega \mathrm{m}$ the relative error is comparable to the one of the real part, independent of the halfspace resistivity and permittivity. For smaller resistivities, the error increases with decreasing resistivity and permittivity. However, relating this behaviour to the absolute values of the imaginary transfer impedance highlights a strong correlation; for resistivities smaller than $5 \mathrm{k} \Omega \mathrm{m}$ the imaginary part of the transfer impedance becomes small $(<10 \mathrm{Ohm})$, but the error large $(>30 \%)$. This suggests that numerical inaccuracies are the cause of the deviations.

Halfspace relative permittivityvs measurement frequency. In Figure 10 the discrepancy between FS and PP solution is shown for a range of halfspace permittivities and measurement frequencies. The absolute values are not shown here, since frequency and permittivity affect the transfer impedance only marginally. For $\operatorname{Re}(\mathrm{Z})$, the discrepancy between FS and PP solutions is hardly affected by these parameters and remains small $(<0.1 \%)$ throughout the investigated $\varepsilon$-f space. These results shows that whilst the permittivity increases the polarizability of the material, this effect is well described by the quasi-static theory for the real part, and relative errors between FS and PP are small. However, $\operatorname{Im}(Z)$ shows again more variability, whereas the relative error is more sensitive to the measurement frequency than to the halfspace permittivity. With increasing frequency and permittivity the relative error is decreasing. 
In summary, the parameter study suggests that the approximation of sensors of finite size by a point pole approximation is valid for $\operatorname{Re}(Z)$, as long as the sensor separation is not smaller than twice the sensor size. Sensor elevation was shown to affect the simulations to a lesser degree. The parameter study indicated a better performance of the CRI technique on resistive ground (> $300 \Omega \mathrm{m}$ ), where relative errors, especially for $\operatorname{Im}(Z)$, generally tended to be small. In general however, $\operatorname{Im}(Z)$ is likely to be more affected by numerical instability. Simulations exploring a range of measurement frequencies indicated a negligible effect on $\operatorname{Re}(Z)$ and only minor effects on $\operatorname{Im}(Z)$ for the discrepancy between FS and PP. It was also shown that the quasi-static theory incorporates the polarizability of the studied material and that the real component of the analytical transfer impedance presents a very good approximation for the simulated one across much of the investigated parameter space. . These results justify the use of $\operatorname{Re}(\mathrm{Z})$ in quasi-static approximation for all data processing. However, the discrepancies between numerical and analytical solutions for $\operatorname{Im}(Z)$ were shown to be strongly dependent on the geometric configuration (i.e. sensor size, separation, elevation) and the material properties (i.e. resistivity, permittivity). Since $\operatorname{Im}(\mathrm{Z})$ for the analytical solution is known to be very sensitive to the geometric setup and resistivities (Kuras et al., 2006; Tabbagh et al., 1993), and the results for the numerical simulations show a similar behaviour, its interpretability will be limited, especially on conductive ground. However, within these studies the highest relative errors in $\operatorname{Im}(\mathrm{Z})$ between FS and PP were often found for parameter combinations where the absolute value of the transfer impedance was comparatively small. This may suggest that some of the large discrepancies are caused by numerical instability, which was found to be more pronounced in $\operatorname{Im}(Z)$ than in $\operatorname{Re}(Z)$. But other cases indicate that large errors are not necessarily related to small absolute values of $\operatorname{Im}(\mathrm{Z})$.

\section{Finite Size Rock Sample}


Building on our experience with the homogeneous halfspace model, a capacitive sensor array used in a real laboratory experiment (Kuras et al., 2012) was simulated in order to validate the measurement principle and to improve our understanding of the measured complex signal. A homogeneous rock sample instrumented with 128 sensors (64 source and 64 potential poles) has been modelled. The sample size is $0.3 \mathrm{~m} \times 0.3 \mathrm{~m} \times 0.45 \mathrm{~m}$, and different electrical resistivities and permittivities can be assigned to the uniform sample domain. Sensors are modelled as plain squares of side length $0.05 \mathrm{~m}$, with an elevation of $0.001 \mathrm{~m}$. This value is at least one order of magnitude higher than realistic sensor elevations achieved in the experiments, but we found that smaller elevations could not be implemented due to the limitations associated with FE mesh generation for the large difference in spatial scales. However, as the parameter study over the homogeneous halfspace has shown, the precise sensor elevation is not necessarily a critical parameter for CRI measurements, provided that the sensors are close to the surface (relative to their size). This is particularly true for $\operatorname{Re}(\mathrm{Z})$, which largely remains constant for small elevations $(<0.03 \mathrm{~m})$. Once again the "Terminal"-Boundary condition was assigned to the source domains.. Between the electrode and sample domains, thin sheets with the dielectric properties of acetate $\left(\sigma \sim 0 \mathrm{~S} / \mathrm{m}, \varepsilon_{\mathrm{r}}=4(\right.$ Wood and Brobst, 1932)) represent the insulation layer as used in the laboratory setup. The complete geometry is placed within a larger domain $(5 \mathrm{~m} \times$ $5 \mathrm{~m} \times 6 \mathrm{~m}$ ) that accounts for the potential distribution in air around the sample. Analogous to the homogeneous halfspace model, at the outer model boundaries domains were defined comprising infinite elements in order to reduce boundary effects. The model layout as implemented in Comsol together with a typical discretisation of the sample domain is shown in Figure 11. Due to the use of unstructured meshes all edges of the sample are very finely discretised, since these are areas of the model which may cause strong effects on the potential distribution.

Since the aim of this simulation was to model sequential 3D CRI measurements across a large set of source-receiver combinations (2408 different combinations), an automatic change 
of source and receiver domains had to be implemented for the FE model. A "parametric sweep" (i.e. an automated, sequential change of model parameters) was defined, which ensured repeated solving of the forward problem for different source dipoles. For each solution the potentials at every receiving sensor were calculated and saved. After the solution of the complete set of source-receiver combinations, values corresponding to the chosen dipole sequence were extracted. Calculation time for a complete set of 2408 different dipole combinations (768 different source dipoles) on a mesh consisting of 1,171,057 elements and 1,636,819 degrees of freedom was about 12 hours (on a computer consisting of 4 Intel ${ }^{\circledR}$ Xeon ${ }^{\circledR}$ X7560 processors with 8 cores each and 128 GB memory). The stability of the solution was tested by calculating a subset of the source sequence on finer meshes. Within the numerical accuracy (i.e. double precision), the results were found to be identical. Moreover, the solutions for separate source dipoles converged rapidly in a monotonical fashion, suggesting that the problem is well posed.

Figure 12 shows our workflow for setting up a 3D model to simulate sequential CRI measurements. Important for the sequential solution of the model is the definition of at least 2 variables (e.g. dom_C1, dom_C2) representing the source domains (one charged positive, one negative). These variables can be used to dynamically define a source current on arbitrary sensors by means of a logical (Boolean) comparison with the Comsol-internal definition of an active domain, called "dom" (e.g. dom == dom_C1). Using a parametric sweep, we were able to change the value of dom_C1 and dom_C2, and thus the current source domain, after each solution of the model. Moreover, defining variables for other model properties (e.g. sample resistivity, permittivity, and frequency) allowed us to carry out additional parameter studies. Due to the complexity of the model (and the large contrasts in modelled scale) we decided to define a number of nested regions for refined discretisation, for example around the sample and sensors (Figure 11). Once the simulation of the complete set of source dipoles had finished, integrals over all sensor domains representing potential poles were calculated to obtain the corresponding potentials at the receiving sensors. By extracting source and receiver 
combinations according to a predefined set, the simulated values could then easily be compared to experimentally measured data.

Simulation Results. In order to compare the simulations with experimentally obtained data, the model was solved for different sample resistivities ( $\rho=85 \Omega \mathrm{m}, 150 \Omega \mathrm{m}, 295 \Omega \mathrm{m}$, and $555 \Omega \mathrm{m})$ and measurement frequencies $(f=15 \mathrm{kHz}$, and $50 \mathrm{kHz})$. A solution of the model using $\rho=555$ $\Omega \mathrm{m}$ and $f=50 \mathrm{kHz}$ is shown in Figure 13 .

The simulated dipole configurations comprise three different types of electrode arrays: (i) a "cross-sample" (CS) array analogous to conventional horizontal and diagonal crossborehole arrays as shown in Chambers et al., 2007; (ii) an "over-edge" (OE) array distributing the dipoles over two adjacent faces; and (iii) a "same-face" (SF) array placing the four poles on the same face, similar to a Wenner $\gamma$-type. Figure 14 shows examples of the array geometries employed for the simulations.

The solutions for all combinations are shown in Figure 13. It is evident that the majority of data points lie in the $4^{\text {th }}$ quadrant of the complex plane (i.e. positive real, negative imaginary part) and that all data points are aligned along the same axis through the origin; this axis is inclined against the $x$-axis by a small phase angle $\left(\varphi \approx 0.92^{\circ}\right)$. This observation compares well with previous experimental data, where all data points lay in the same $\left(4^{\text {th }}\right)$ quadrant. A small phase angle of $\varphi \approx 1^{\circ}$ was expected; reflecting the fact that the quadrature component of the signal is very small compared to the in-phase component. This observation is compatible with the quasi-static theory (Kuras et al., 2006) as discussed in the description of the analytical solution. It provides further justification for using only the in-phase component $(\operatorname{Re}(\mathrm{Z}))$ to derive apparent resistivities.

If we consider the CS array only (blue markers in Figure 13) then all data points are found to lie in the $4^{\text {th }}$ quadrant, which is also the expected result for a 1D situation. For the OE array, the distribution of data points shows most of them in the $4^{\text {th }}$ quadrant, but also spans over 
the $2^{\text {nd }}$ and $3^{\text {rd }}$ quadrant (negative real, positive and negative imaginary part, respectively). In general, the distribution is broader than for the CS array. In contrast to the OE array, the SF array shows only a few data points outside of the $4^{\text {th }}$ quadrant, namely in the $1^{\text {st }}$ and $2^{\text {nd }}$ quadrant (positive and negative real, positive imaginary part, respectively), but more scattering in the data is observed. Figure 13 also gives an indication of the dc geometric factors for the data points shown. The contour plot suggests that data points in the $2^{\text {nd }}$ and $3^{\text {rd }}$ quadrant are associated with negative geometric factors, which are characteristic for some of the unusual sensor geometries of the OE array.

If acquisition is limited to all possible dipole combinations of the CS array only (this array tends to produce the least noisy measured data), the measurement sequence should still be able to recover the spatial characteristics of the resistivity distribution across the whole rock sample and provide good quality data at the same time. As a consequence, this type of sensor arrangement is likely to present the best compromise for practical 3D resistivity imaging of a rock sample. However, we are conscious that our results reflect homogeneous sample properties only. The sensitivity of different array geometries to heterogeneities in sample resistivity would have to be evaluated in order to assess this aspect quantitatively. An optimal set of dipole combinations for high-resolution imaging of such a rock sample could then be determined as a function of the specific resistivity distribution.

Investigation of the voltage distribution on the sample suggests that potential sensors in the immediate vicinity of the source poles may experience the near field of the induced potential, where comparatively large voltage contrasts can occur on the scale of the sensor dimensions. This observation may explain the results obtained for the homogeneous halfspace model, which suggested that with decreasing sensor separation the point-pole approximation breaks down and larger geometric errors must be expected. However, our implementation of the 3D model does 
not account for the potential sensors being finite-size metallic conductors that will distort the resulting electrical field; in reality the sensors will represent an equipotential surface.

Solving the model for different sample resistivities confirmed the linear relationship between sample resistivity and the simulated $\operatorname{Re}(Z)$. This direct relationship is the fundamental prerequisite for resistivity imaging with capacitive sensors. The results for the imaginary part showed only very subtle changes associated with increasing sample resistivities. However, the magnitude of $\operatorname{Im}(Z)$ is much smaller than that of $\operatorname{Re}(Z)$. Changing the measurement frequency from $15 \mathrm{kHz}$ to $50 \mathrm{kHz}$ showed no effects on the simulated $\mathrm{Re}(\mathrm{Z})$ at the investigated sample resistivity, but it has to be noted that higher frequencies and sample resistivities would also alter the real part of the transfer impedances; as demonstrated for the homogeneous halfspace model.

\section{Comparison with measured data}

The simulations of CRI measurements on a finite rock sample were inspired by the work of Kuras et al. (2012), where CRI measurements are used to monitor the physical modelling of permafrost processes in chalk and limestone. These experiments define the requirements for the sensor and sample arrangement in our models, which makes comparison between simulated and measured data straightforward. For the purpose of this comparison, the median resistivity for the experimental data sets (i.e. using the data acquired over the whole sample) was used to define the (homogeneous) sample resistivity in the numerical models.

Figure 15 shows the comparison for $\operatorname{Re}(\mathrm{Z})$ obtained from simulation and experiment for a saturated sample with a median resistivity of $\rho=150 \Omega \mathrm{m}$. The agreement between simulation and experiment is very good, bearing in mind that the simulation assumes a perfectly homogeneous sample with well-defined sensor-sample separation and sample geometry, whereas in reality the sample is expected to include heterogeneities and its geometric shape is 
imperfect. The simulated $\operatorname{Re}(\mathrm{Z})$ lies in the range of 25 to $1350 \Omega$, which compares well with the measured data (10 to $1400 \Omega$ ). The high logarithmic correlation coefficient $r=0.953$ indicates good positive correlation between the two data sets, which is also reflected in a very reasonable alignment of the data along the diagonal. Differences between simulation and experiment increase with increasing impedances, but the relative error remains at the same level (about $10 \%)$ throughout the range of impedances. Outliers are usually related to data points where the measured imaginary component is larger than the simulated one, i.e. where the logarithmic ratio of measured to simulated $\operatorname{Im}(Z)$ is greater than one (warm colours in Figure 15). This observation may offer an opportunity to use the imaginary part to evaluate the quality of CRI measurements. Furthermore, these results validate our approach to simulating CRI measurements numerically, and they prove the applicability of the methodology to the imaging of laboratory-scale samples.

\section{Conclusions}

In this paper we used numerical simulations with the aim of improving our understanding of practical CRI measurements and to define limitations on the array design, based on the assessment of relative errors between geometrically realistic simulation results and analytical solutions, derived from a point pole approximation. Simulations of CRI measurements above a homogeneous halfspace showed that the real component of the complex transfer impedance depends mainly on sensor size and separation; the smaller the normalised sensor separation the larger the discrepancy between the simulated and the analytical solutions. The point-pole approximation of the analytical solution breaks down if the sensor separation is smaller than 4 times the sensor size. To avoid large errors (> 5\%), the sensors have to be separated by more than twice their size. The influence of sensor elevation, halfspace resistivity, halfspace permittivity, and measurement frequency was investigated and their effect on the relative error between simulation and analytical solution was shown to be negligible for the case of $\operatorname{Re}(\mathrm{Z})$. It 
was also shown that the imaginary component is more sensitive to the geometric configuration of the sensors (i.e. normalised sensor separation and elevation) and to measurement and dielectric ground properties (i.e. measurement frequency, halfspace resistivity, and permittivity) than $\operatorname{Re}(Z)$. Therefore, opportunities for the quantitative interpretation of $\operatorname{Im}(Z)$ are limited. Additionally, it was highlighted that $\operatorname{Im}(\mathrm{Z})$ is more susceptible to numerical instability in the models than $\operatorname{Re}(\mathrm{Z})$.

In order to prove the applicability of CRI simulations to experimental studies, we simulated sequential CRI imaging measurements on a 3D finite rock sample and compared them to measured data, thus fully exploiting the capabilities of finite-element numerical modelling. A comparison of the two data sets showed good agreement and validated the obtained results. More fundamentally, this outcome demonstrates that experimental CRI measurements can be simulated with a finite element approach using a quasi-static regime, as results obtained are in accord with experimental data. Additionally, we were able to associate outliers in the experimental data with large discrepancies between simulated and measured $\operatorname{Im}(\mathrm{Z})$, thus highlighting the benefit of simulating CRI measurements in terms of data quality assessment. We showed that the use of finite-element numerical simulations of CRI measurements is a valuable tool to define limitations on array design and to evaluate measurement quality.

\section{Acknowledgement}

Our work is funded by a Natural Environment Research Council (NERC) Technology Proof of Concept grant award (NE/I000917/1). The research formed part of Sebastian Uhlemann's M.Sc. dissertation at ETH Zurich, department of applied and environmental geophysics, under cosupervision of Prof. Alan Green. We thank Andreas Hördt, an anonymous reviewer and the associate editor, for their fruitful comments, which have greatly improved an earlier version of the manuscript. This paper is published with the permission of the Executive Director of the British Geological Survey (NERC). 


\section{References}

Benderitter Y, Jolivet A, Mounir A, Tabbagh A. 1994. Application of the electrostatic quadripole to sounding in the hectometric depth range. Journal of Applied Geophysics 31 : 1-6.

Butler SL, Sinha G. 2012. Forward modeling of applied geophysics methods using Comsol and comparison with analytical and laboratory analog models. Computers \& Geosciences 42 : 168176.

Chambers JE, Wilkinson PB, Weller AL, Meldrum PI, Ogilvy RD, Caunt S. 2007. Mineshaft imaging using surface and crosshole 3D electrical resistivity tomography: A case history from the East Pennine Coalfield, UK. Journal of Applied Geophysics 62 : 324-337.

COMSOL. 2011. COMSOL Multiphysics User's Guide, Version 4.2

Grard R. 1990. A quadrupolar array for measuring the complex permittivity of the ground: application to Earth prospection and planetary exploration. Measurement Science and Technology 1 : 295-301.

Grard R, Tabbagh A. 1991. A mobile four-electrode array and its application to the electrical survey of planetary grounds at shallow depths. Journal of Geophysical Research 96 : 4117 4123.

Hördt, A, Weidelt, P, Przyklenk, A. 2013. Contact Impedance of Grounded and Capacitive Electrodes. Geophysical Journal International 193 : 187-196. 
Krautblatter M, Hauck C. 2007. Electrical resistivity tomography monitoring of permafrost in solid rock walls. Journal of Geophysical Research 112 : 1-14.

Krautblatter M, Verleysdonk S, Flores-Orozco A, Kemna A. 2010. Temperature-calibrated imaging of seasonal changes in permafrost rock walls by quantitative electrical resistivity tomography (Zugspitze, German/Austrian Alps). Journal of Geophysical Research 115 : 1-15.

Kuras O, Beamish D, Meldrum PI, Ogilivy, RD . 2006. Fundamentals of the capacitive resistivity technique. Geophysics 71 : G135-G152.

Kuras O, Krautblatter M, Murton JB, Haslam E, Meldrum PI, Wilkinson PB, Uhlemann SS. 2012. Monitoring Rock-freezing Experiments in the Laboratory with Capacitive Resistivity Imaging, Near Surface 2012, Paris, France, Extended Abstracts. A06.

Marescot L, Rigobert S, Palma Lopes S, Lagabrielle R, Chapellier D. 2006. A general approach for DC apparent resistivity evaluation on arbitrarily shaped 3D structures. Journal of Applied Geophysics 60 : 55-67.

McNeill JD. 1980. Electromagnetic terrain conductivity measurement at low induction numbers . Geonics Limited

Reynolds, JM. 2011. An Introduction to Applied and Environmental Geophysics, 2nd edn, pp.293-295 . John Wiley \& Sons Ltd, Chichester

Tabbagh A, Hesse A, Grard R. 1993. Determination of Electrical Properties of the Ground at Shallow Depth with an Electrostatic Quadrupole: Field Trials on Archaeological Sites.

Geophysical prospecting $41: 579-597$.

Wood EB, Brobst DR. 1932. Cellulose Acetate Treatment for Textile Insulation - Engineering Development. Bell System Technical Journal $11: 213-230$. 


\section{List of captions}

Table 1 Values of the induction number B for best to worst case scenarios using typical values used in the numerical simulations. It shows that the B clearly remains below 1 for all investigated scenarios.

Figure 1 Schematic drawing of capacitively coupled qadrupole, non-grounded in air. $C_{1}$ and $C_{2}$ are source poles, $\mathrm{P}_{1}$ and $\mathrm{P}_{2}$ are potential poles, and $\mathrm{C}^{\prime} 1$ and $\mathrm{C}_{2}^{\prime}$ are image source poles. Indicated are distances between potential and source or image source points r or r', respectively. The first subscript indicates the potential pole and the second the source pole.

Figure 2 Dependence of complex transfer impedance on sensor elevation, displayed for five different halfspace resistivities. Dipole separation $r=1 \mathrm{~m}, \varepsilon_{\mathrm{r}}=1, \mathrm{f}=15 \mathrm{kHz}$. Please note that all sensors are assumed to have exactly the same elevation.

Figure 3 Geometric configuration (left) and example mesh (right) used in the parameter study of a capacitively coupled quadrupole over a homogeneous halfspace. Infinite elements are assigned to surrounding outer boundary domains as indicated.

Figure 4 Surface plots showing (top) the range of absolute values of the real and imaginary transfer impedances from the analytical solution, (bottom) the discrepancies between simulated complex transfer impedance and analytic solution for different normalised sensor separations and halfspace resistivities (w $=0.05 \mathrm{~m}, \mathrm{~h}=0.001 \mathrm{~m}, \varepsilon=3$, and $\mathrm{f}=15 \mathrm{kHz})$.

Figure 5 Surface plots showing (top) the range of absolute values of the real and imaginary transfer impedances from the analytical solution, (bottom) the discrepancy between simulated complex transfer impedance and analytic solution for different sensor elevations and halfspace resistivities $(\mathrm{w}=0.05 \mathrm{~m}, \mathrm{~h}$

$=0.001 \mathrm{~m}, \varepsilon=3$, and $\mathrm{f}=15 \mathrm{kHz})$.

Figure 6 Surface plots showing (top) the absolute values of the complex transfer impedance and (bottom) discrepancy between simulated complex potential and analytic solution for 
combinations of sensor elevation and normalised sensor separation $(\mathrm{w}=0.05 \mathrm{~m}, \rho=100 \Omega \mathrm{m}, \varepsilon$ $=3$, and $\mathrm{f}=15 \mathrm{kHz})$.

Figure 7 Surface plots showing the discrepancy between simulated complex potential and analytic solution for different heights of one out of the four poles at a range of halfspace resistivities $\left(h\right.$ (remaining poles) $=0.001 \mathrm{~m}, l_{r}=20, \varepsilon=3$, and $\left.f=15 \mathrm{kHz}\right)$.

Figure 8 Surface plots showing (top) the absolute values of the complex transfer impedance and (bottom) discrepancy between simulated complex potential and analytic solution for combinations of measurement frequency and halfspace resistivity $\left(h=0.001 \mathrm{~m}, l_{r}=20\right.$, and $\varepsilon=$ 3) . .46

Figure 9 Surface plots showing (top) the absolute values of the complex transfer impedance and (bottom) discrepancy between simulated complex potential and analytic solution for combinations of halfspace permittivity and halfspace resistivity $\left(h=0.001 \mathrm{~m}, l_{r}=20\right.$, and $f=15$ $\mathrm{kHz})$.

Figure 10 Surface plots showing the discrepancy between simulated complex potential and analytic solution for different halfspace permittivities at a range of halfspace resistivities $(h=$ $0.001 \mathrm{~m}, l_{r}=20$, and $\left.\rho=100 \Omega \mathrm{m}\right)$.

Figure 11 Geometric configuration (left) and example mesh (right) used in the simulation of the laboratory experiments.

Figure 12 Workflow for setting up a sequential simulation of CRI measurements in Comsol Multiphysics ${ }^{\circledR}$ 50

Figure 13 Complex transfer impedance calculated by simulating sequential CRI measurements on a finite rock sample of $\rho=555 \Omega \mathrm{m}, \varepsilon_{\mathrm{r}}=2.4$, using $\mathrm{f}=50 \mathrm{kHz}$ (note the different scale of the $\mathrm{y}$-axis) 51

Figure 14 Electrode arrays employed in the numerical simulation. a) cross-sample CS; b) over-edge OE; c) same-face SF. 
Figure 15 a) Comparison of the real part of the simulated and experimentally obtained transfer impedances for a saturated sample with mean resistivity of $150 \Omega \mathrm{m}$. Note the logarithmic correlation factor of $r=0.953$. Data points are coloured using the logarithmic of the ratio between measured and simulated imaginary part of the transfer impedance. b) Distribution of measured apparent resistivities. . 54 
Table 1 Values of the induction number B for best to worst case scenarios using typical values used in the numerical simulations. It is evident that B remains well below 1 for all investigated scenarios.

\begin{tabular}{|ll|ccc|}
\hline & & Low & Mid & High \\
\hline $\mathbf{f}$ & {$[\mathrm{kHz}]$} & 10 & 25 & 150 \\
$\mathbf{L}$ & {$[\mathrm{m}]$} & 0.05 & 0.5 & 1 \\
P & {$[\Omega \mathrm{m}]$} & $10^{5}$ & $10^{3}$ & $10^{1}$ \\
\hline B & & $\mathbf{3 . 1 4 1 6 \cdot 1 0 ^ { - 5 }}$ & $\mathbf{4 . 9 6 7 3 \cdot 1 0 ^ { - 3 }}$ & $\mathbf{0 . 2 4 3 4}$ \\
\hline
\end{tabular}




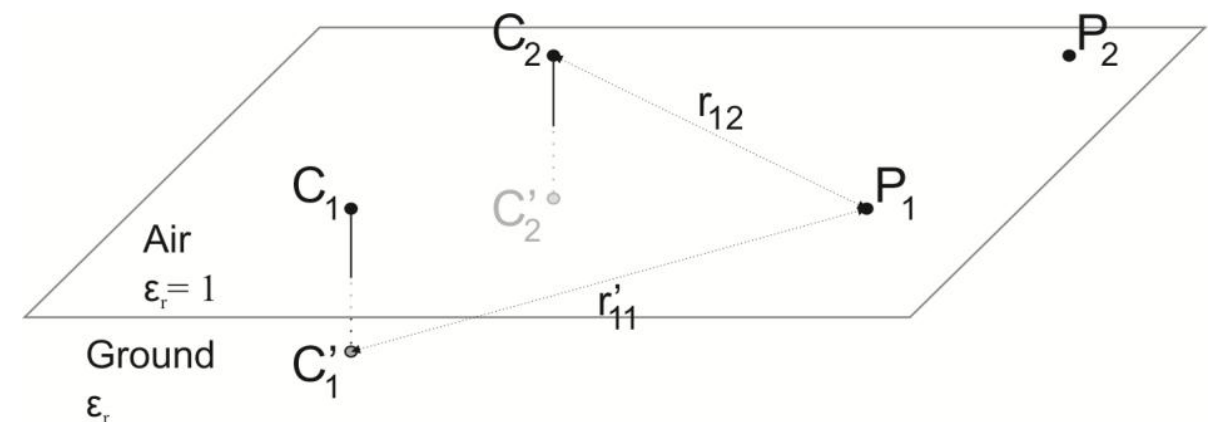

Figure 1 Schematic drawing of capacitively coupled quadrupole, non-grounded in air. $\mathrm{C}_{1}$ and $\mathrm{C}_{2}$ are source poles, $\mathrm{P}_{1}$ and $\mathrm{P}_{2}$ are potential poles, and $\mathrm{C}^{\prime} 1$ and $\mathrm{C}_{2}^{\prime}$ are image source poles.

Indicated are distances between potential and source or image source points $r$ or $r^{\prime}$, respectively. The first subscript indicates the potential pole and the second the source pole. 

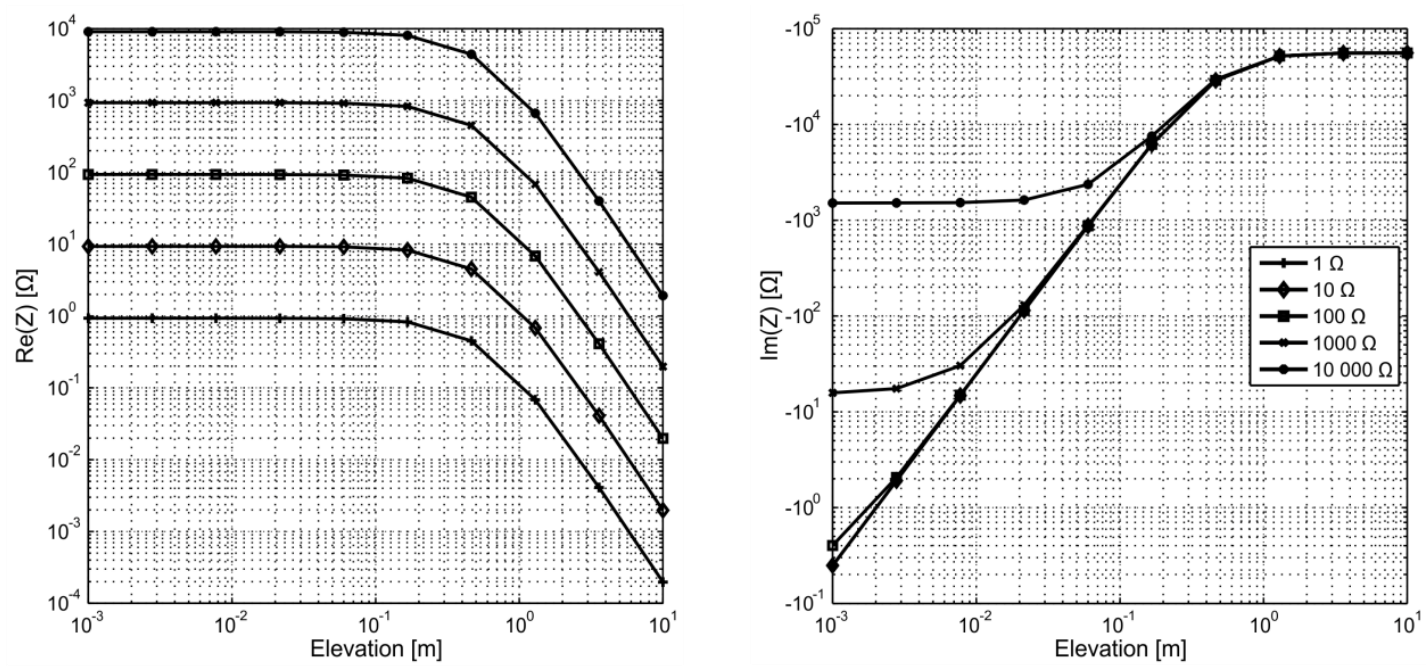

Figure 2 Dependence of complex transfer impedance on sensor elevation, displayed for five different halfspace resistivities. Dipole separation $r=1 \mathrm{~m}, \varepsilon_{r}=1, f=15 \mathrm{kHz}$. Please note that all sensors are assumed to have exactly the same elevation. 


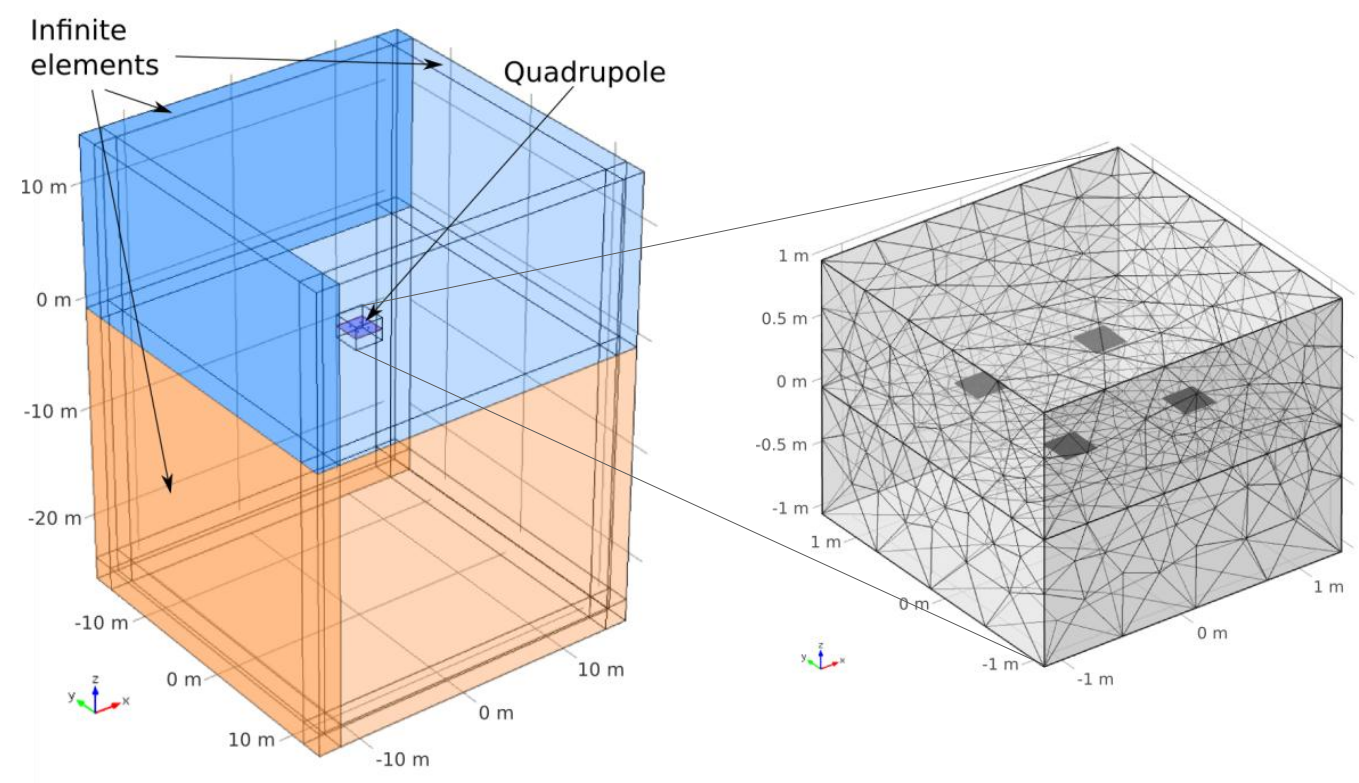

Figure 3 Geometric configuration (left) and example mesh (right) used in the parameter study of a capacitively coupled quadrupole over a homogeneous halfspace. Infinite elements are assigned to surrounding outer boundary domains as indicated. 

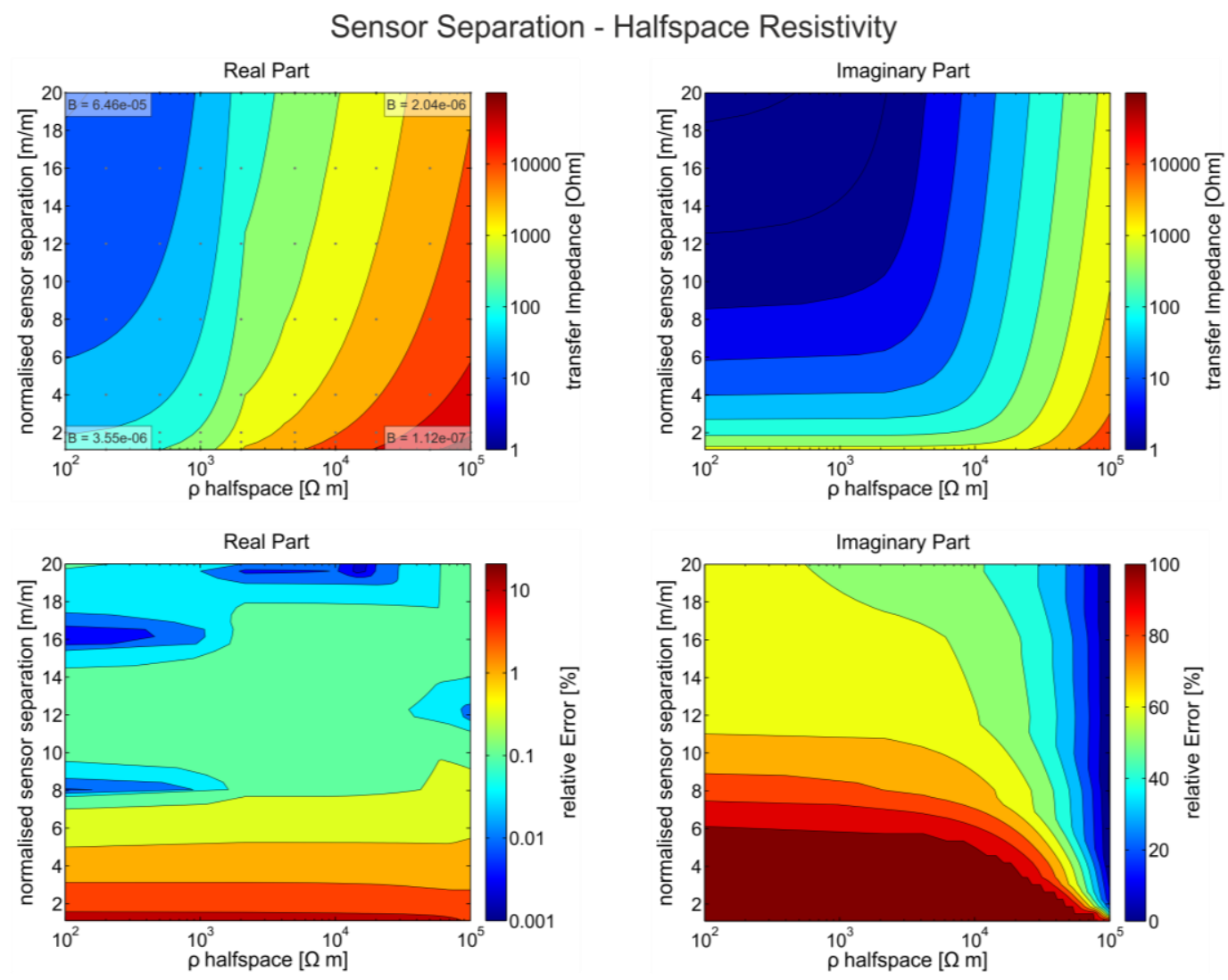

Figure 4 Surface plots showing (top) the range of absolute values of the real and imaginary transfer impedances from the analytical solution, (bottom) the discrepancies between simulated complex transfer impedance and analytic solution for different normalised sensor separations and halfspace resistivities $(w=0.05 \mathrm{~m}, h=0.001 \mathrm{~m}, \varepsilon=3$, and $f=15 \mathrm{kHz})$. 

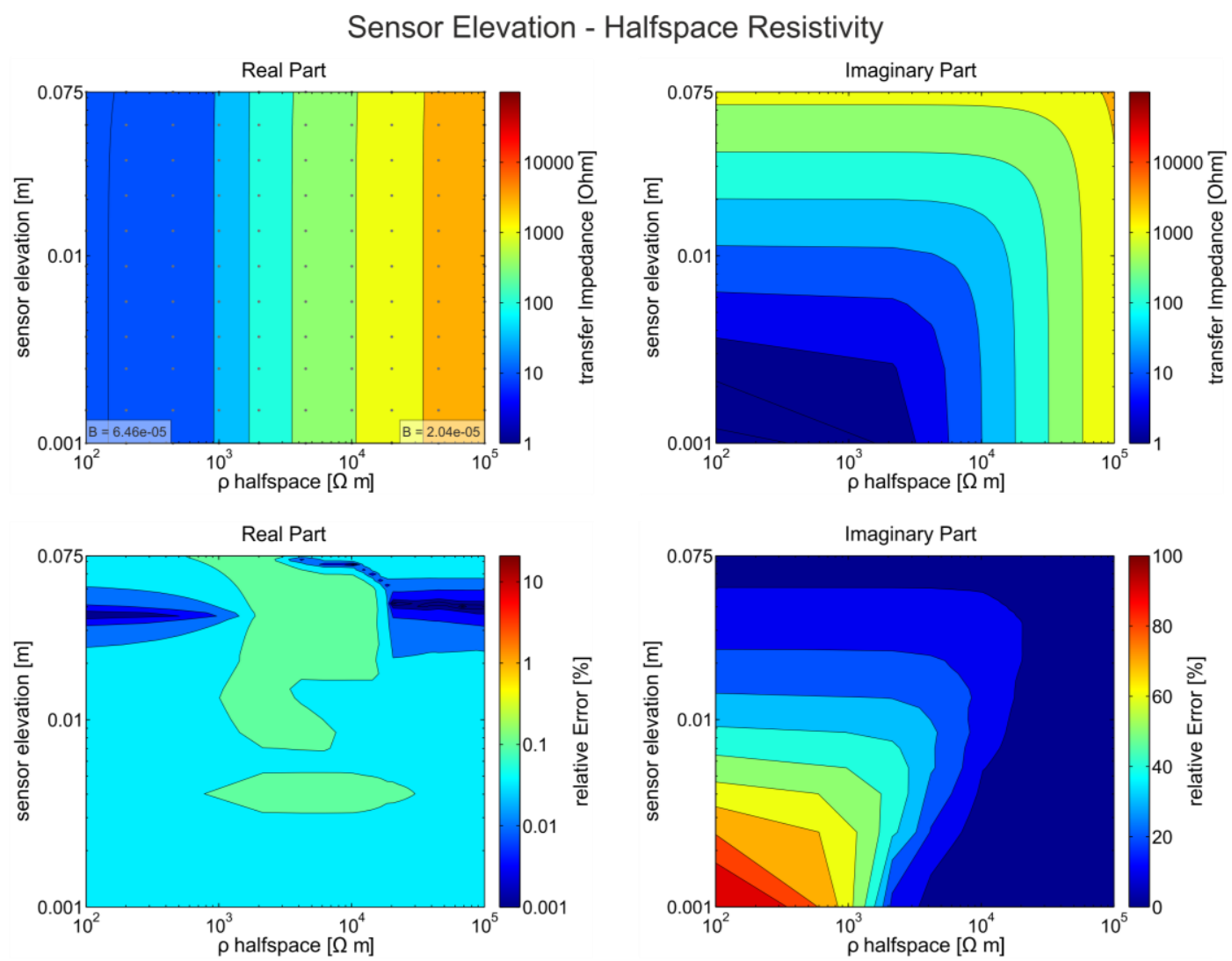

Figure 5 Surface plots showing (top) the range of absolute values of the real and imaginary transfer impedances from the analytical solution, (bottom) the discrepancy between simulated complex transfer impedance and analytic solution for different sensor elevations and halfspace resistivities ( $w=0.05 \mathrm{~m}, h=0.001 \mathrm{~m}, \varepsilon=3$, and $f=15 \mathrm{kHz}$ ). 

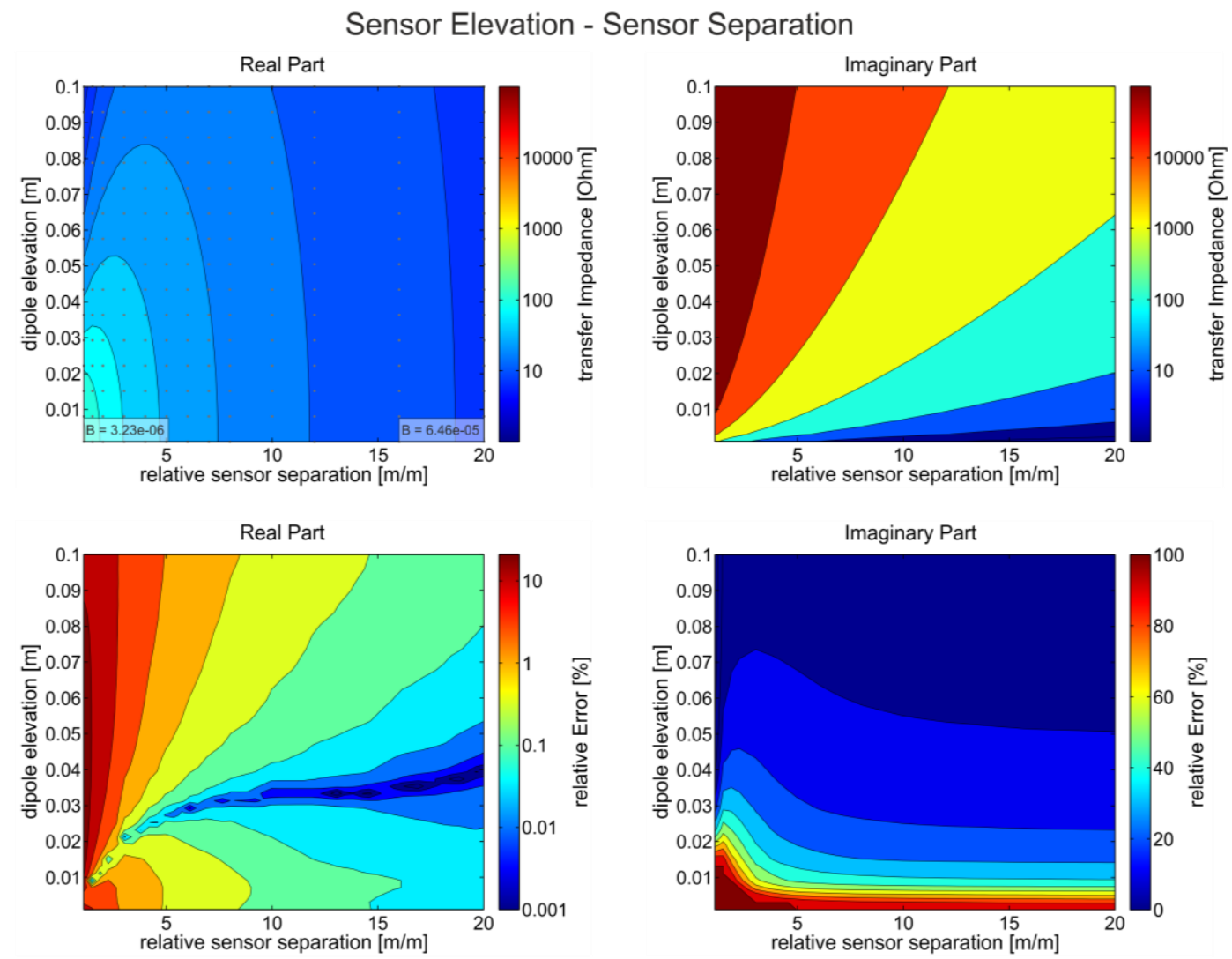

Figure 6 Surface plots showing (top) the absolute values of the complex transfer impedance and (bottom) discrepancy between simulated complex potential and analytic solution for combinations of sensor elevation and normalised sensor separation $(w=0.05 \mathrm{~m}, \rho=100 \Omega \mathrm{m}, \varepsilon$ $=3$, and $f=15 \mathrm{kHz})$. 
Pole Elevation - Halfspace Resistivity
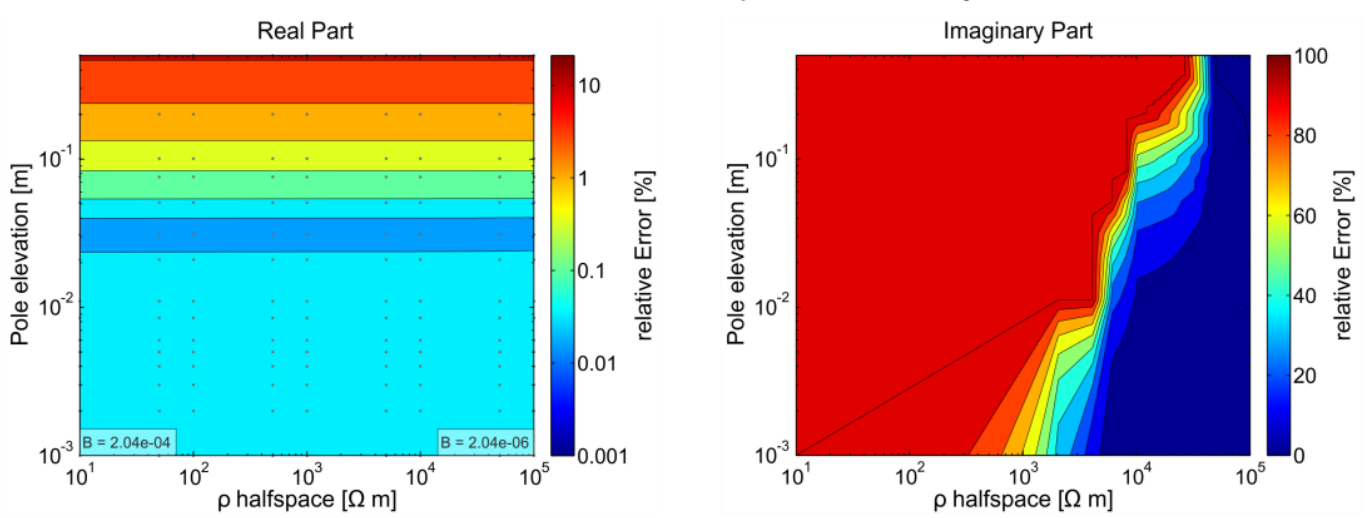

Figure 7 Surface plots showing the discrepancy between simulated complex potential and analytic solution for different heights of one out of the four poles at a range of halfspace resistivities $\left(h\right.$ (remaining poles) $=0.001 \mathrm{~m}, l_{r}=20, \varepsilon=3$, and $f=15 \mathrm{kHz}$ ). 

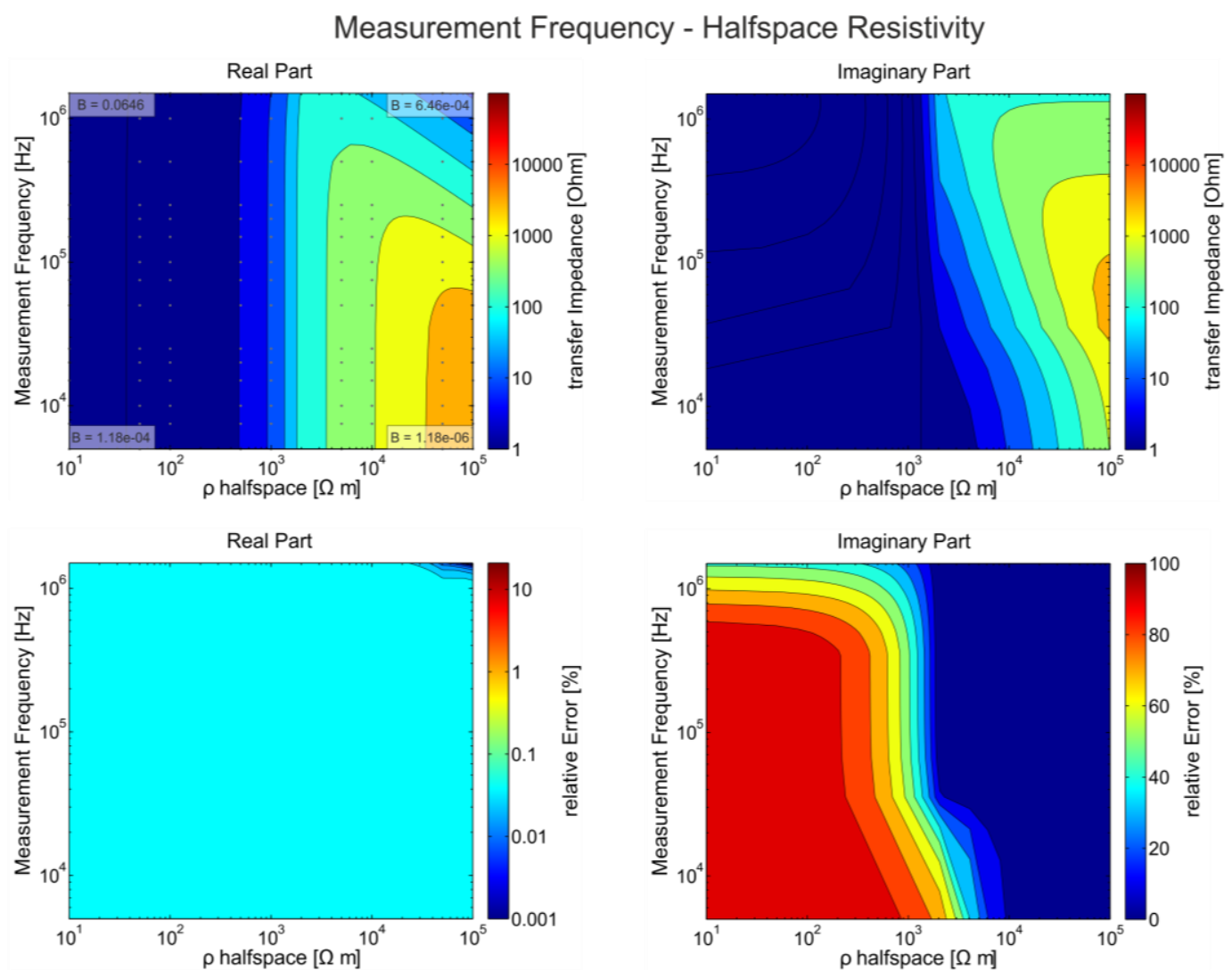

Figure 8 Surface plots showing (top) the absolute values of the complex transfer impedance and (bottom) discrepancy between simulated complex potential and analytic solution for combinations of measurement frequency and halfspace resistivity $\left(h=0.001 \mathrm{~m}, l_{r}=20\right.$, and $\varepsilon=$ $3)$. 

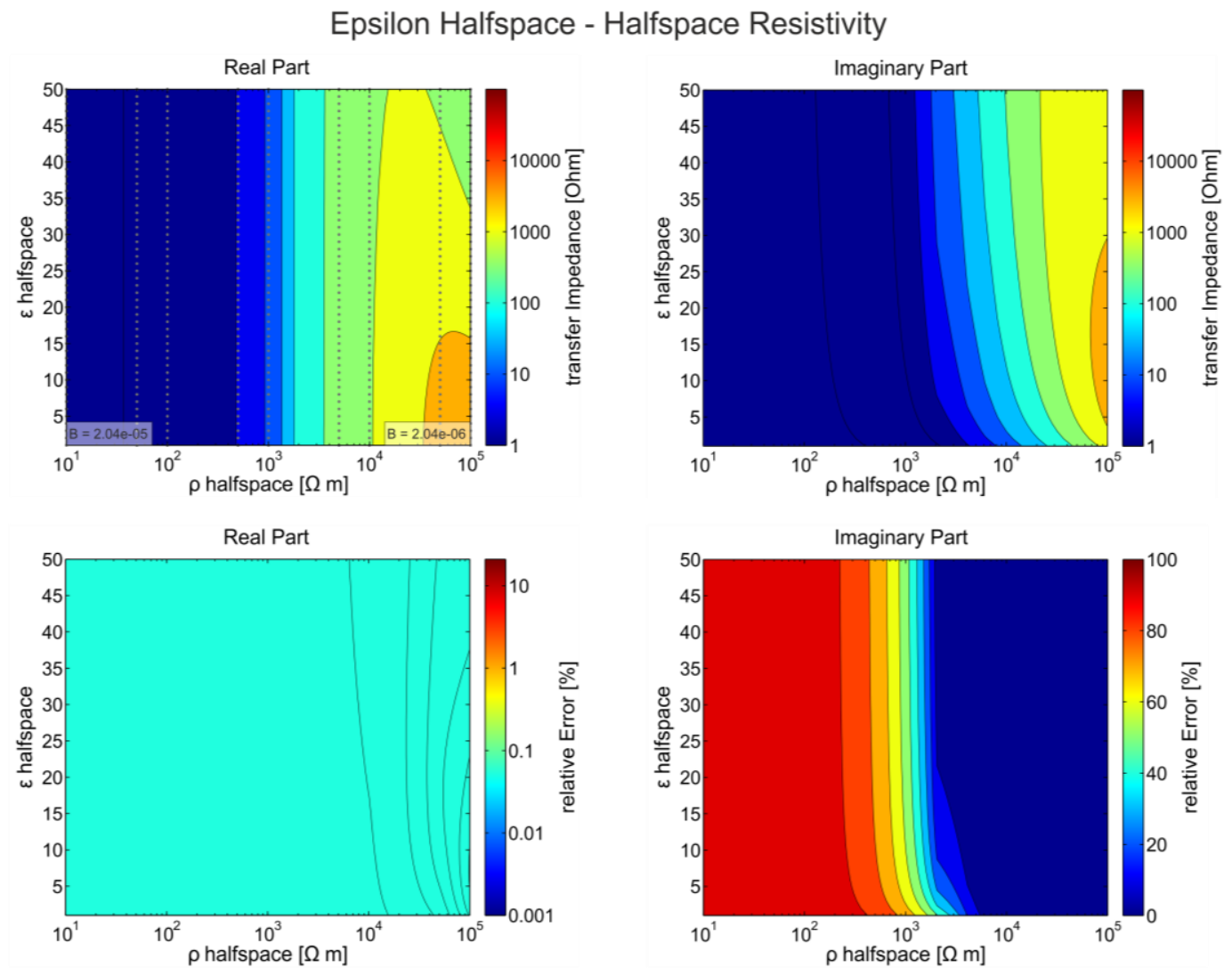

Figure 9 Surface plots showing (top) the absolute values of the complex transfer impedance and (bottom) discrepancy between simulated complex potential and analytic solution for combinations of halfspace permittivity and halfspace resistivity $\left(h=0.001 \mathrm{~m}, l_{r}=20\right.$, and $f=15$ $\mathrm{kHz}$ ). 

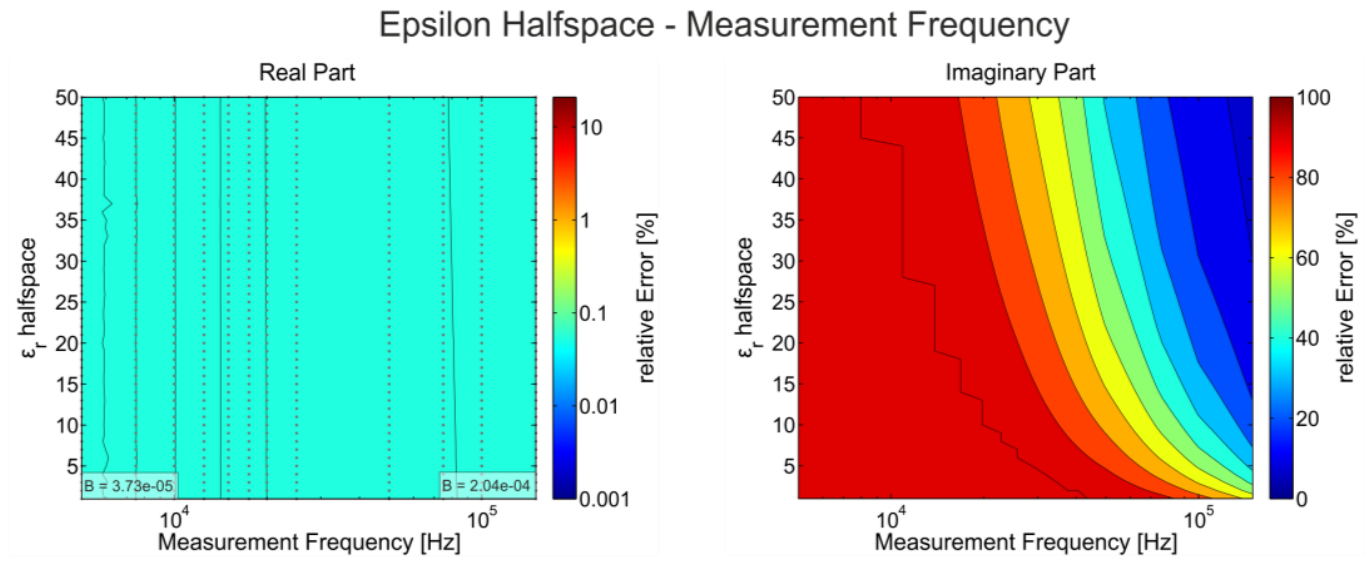

Figure 10 Surface plots showing the discrepancy between simulated complex potential and analytic solution for different halfspace permittivities at a range of measurement frequencies $(h$ $=0.001 \mathrm{~m}, l_{r}=20$, and $\left.\rho=100 \Omega \mathrm{m}\right)$. 


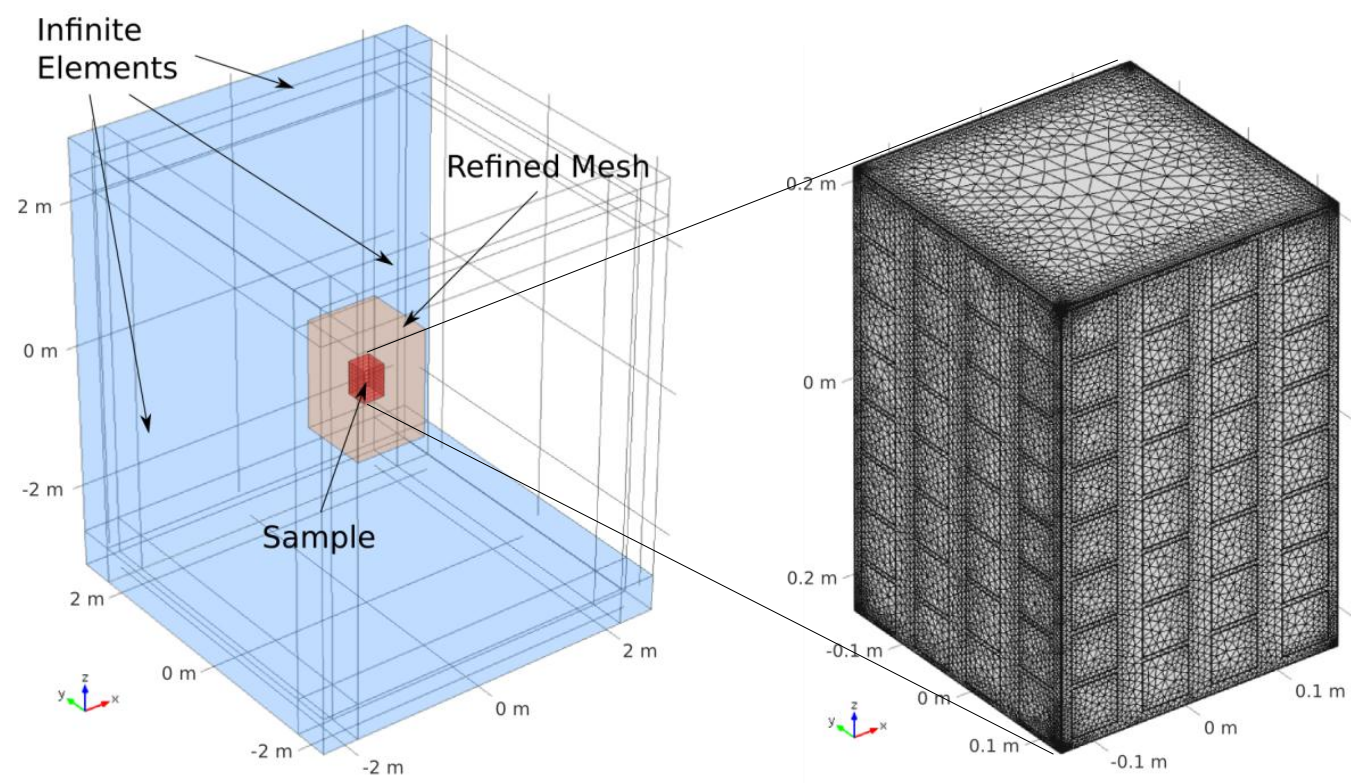

Figure 6 Geometric configuration (left) and example mesh (right) used in the simulation of the laboratory experiments. 


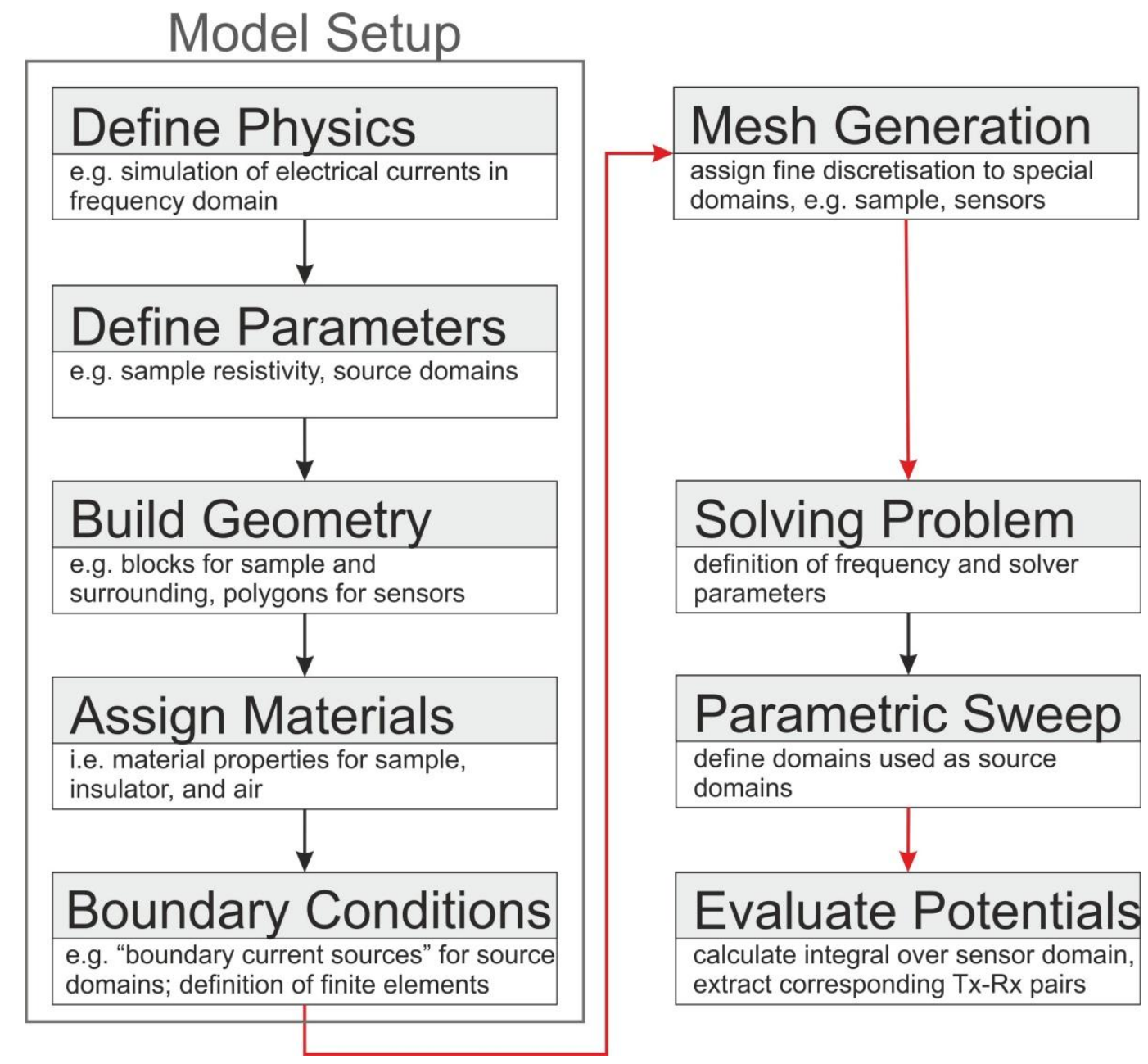

Figure 7 Workflow for setting up a sequential simulation of CRI measurements in Comsol Multiphysics ${ }^{\circledR}$. 


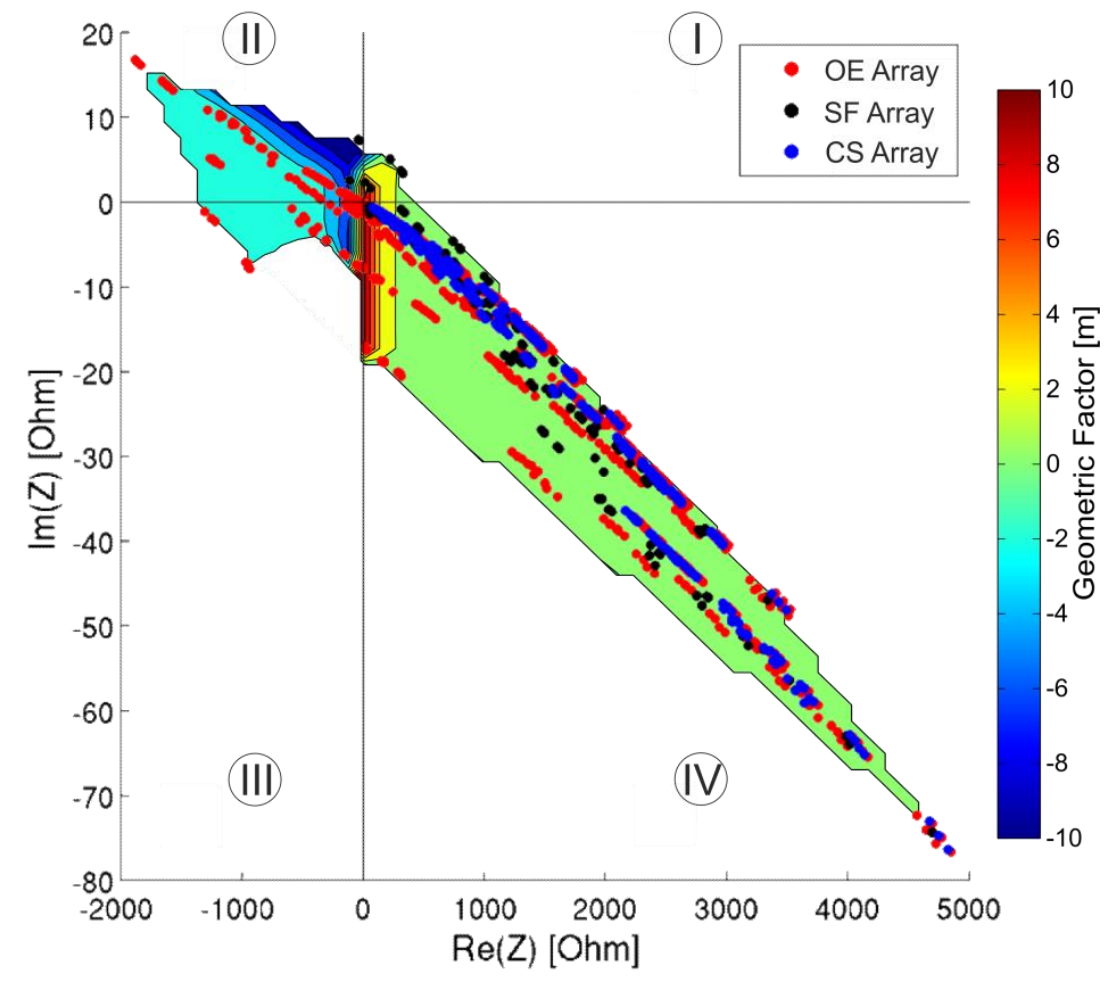

Figure 8 Complex transfer impedance calculated by simulating sequential CRI measurements on a finite rock sample of $\rho=555 \Omega \mathrm{m}, \varepsilon_{r}=2.4$, using $f=50 \mathrm{kHz}$ (note the different scale of the y-axis). 

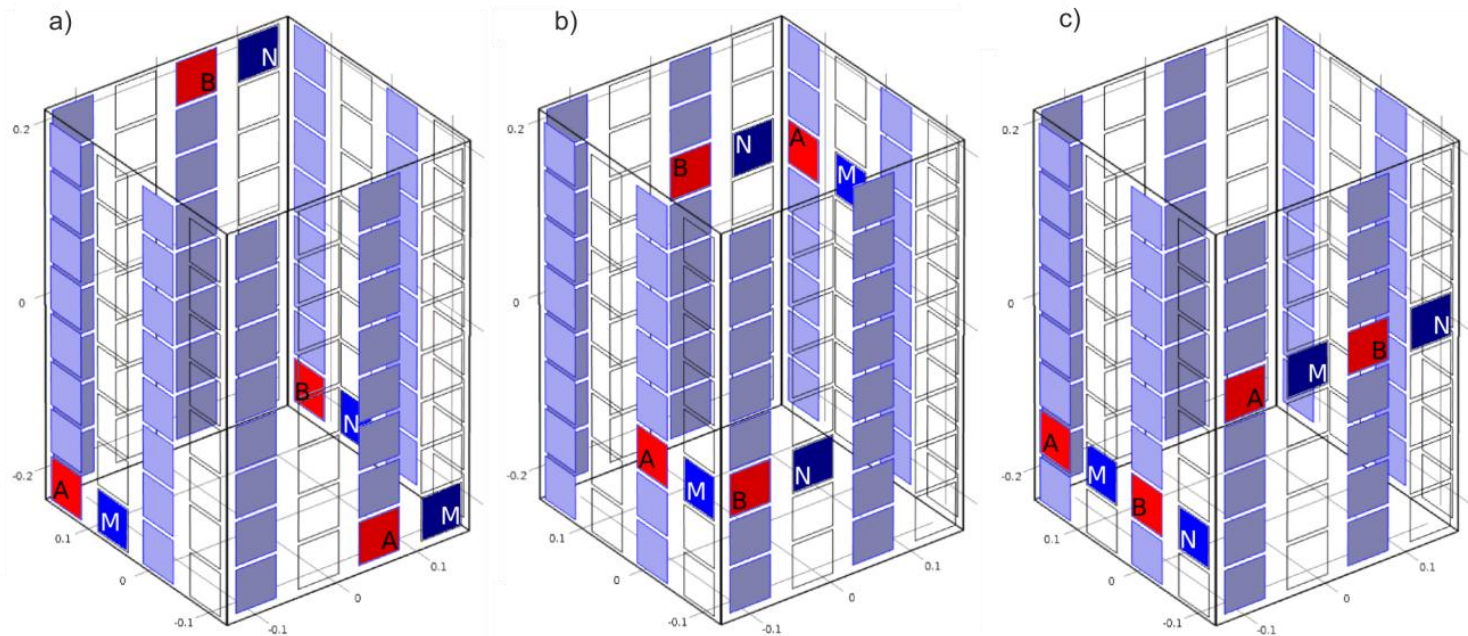

Figure 9 Electrode arrays employed in the numerical simulation. a) cross-sample CS; b) over-edge OE; c) same-face SF. 

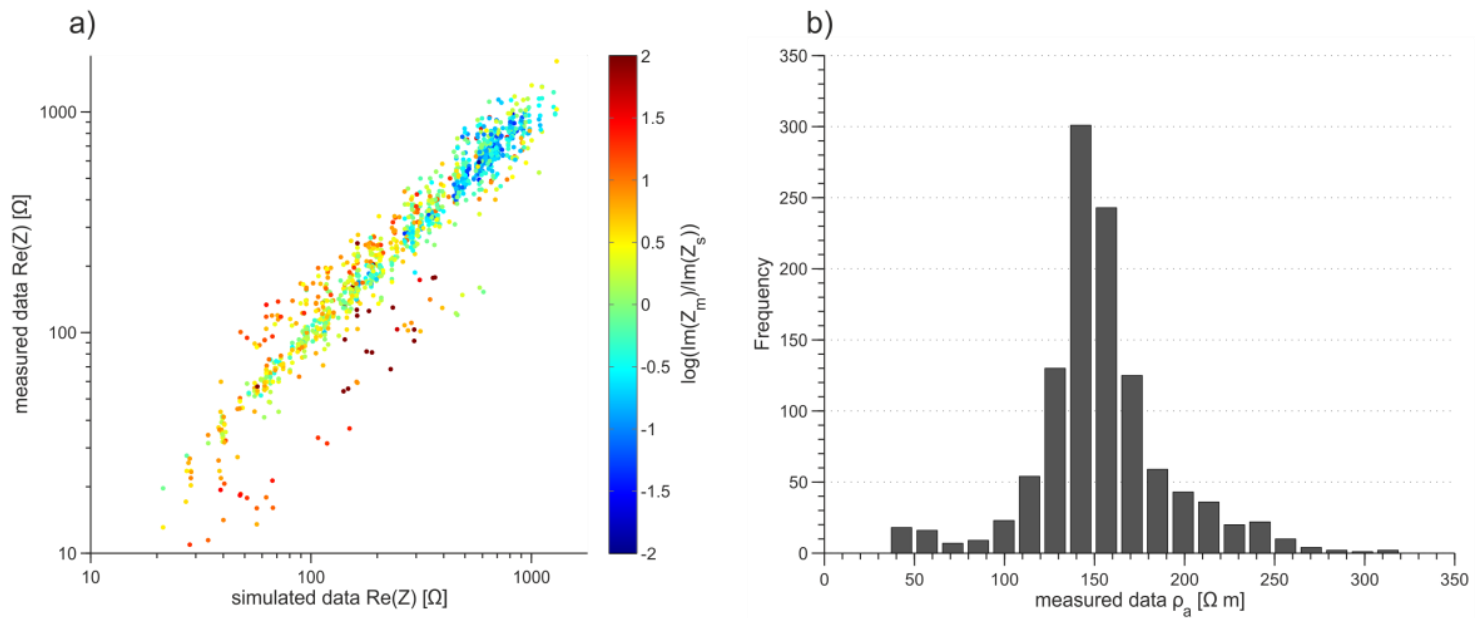

Figure 10 a) Comparison of the real part of the simulated and experimentally obtained transfer impedances for a saturated sample with median resistivity of 150 $\Omega \mathrm{m}$. Note the logarithmic correlation factor of $\mathrm{r}=0.953$. Data points are coloured using the logarithmic of the ratio between measured and simulated imaginary part of the transfer impedance. b) Distribution of measured apparent resistivities. 